\title{
Powerful terahertz waves from long-wavelength infrared laser filaments
}

\author{
Vladimir Yu. Fedorov ${ }^{1,2}$ and Stelios Tzortzakis (1D) ${ }^{1,3,4}$
}

\begin{abstract}
Strong terahertz $(\mathrm{THz})$ electric and magnetic transients open up new horizons in science and applications. We review the most promising way of achieving sub-cycle THz pulses with extreme field strengths. During the nonlinear propagation of two-color mid-infrared and far-infrared ultrashort laser pulses, long, and thick plasma strings are produced, where strong photocurrents result in intense $\mathrm{THz}$ transients. The corresponding $\mathrm{THz}$ electric and magnetic field strengths can potentially reach the gigavolt per centimeter and kilotesla levels, respectively. The intensities of these $\mathrm{THz}$ fields enable extreme nonlinear optics and relativistic physics. We offer a comprehensive review, starting from the microscopic physical processes of light-matter interactions with mid-infrared and far-infrared ultrashort laser pulses, the theoretical and numerical advances in the nonlinear propagation of these laser fields, and the most important experimental demonstrations to date.
\end{abstract}

\section{Introduction}

The terahertz $(\mathrm{THz})$ frequency range is the last lacuna in the electromagnetic spectrum, where we have no efficient sources of radiation. Being usually understood as the region of frequencies from 0.1 to $10 \mathrm{THz}$ or, equivalently, of wavelengths from $3 \mathrm{~mm}$ to $30 \mu \mathrm{m}$, the THz part of the spectrum is squeezed between the domains of highfrequency electronics and photonics. While a large number of powerful microwave sources exist in highfrequency electronics and high-energy lasers cover the needs in photonics, there is currently no easy, direct way to generate $\mathrm{THz}$ fields of any comparable strength.

The $\mathrm{THz}$ frequency range draws attention for a number of reasons. First, $\mathrm{THz}$ waves penetrate almost losslessly through a large number of different materials, such as plastics, fabrics, concrete, wood, and paper. However, unlike X-rays, the energy of $\mathrm{THz}$ photons, being on the order of several $\mathrm{meV}$, is too low to directly disrupt any

Correspondence: Vladimir Yu Fedorov (v.y.fedorov@gmail.com) or

Stelios Tzortzakis (stzortz@iesl.forth.gr)

${ }^{1}$ Science Program, Texas A\&M University at Qatar, P.O. Box 23874, Doha, Qatar

${ }^{2}$ P.N. Lebedev Physical Institute of the Russian Academy of Sciences, 53 Leninskiy Prospekt, Moscow 119991, Russia

Full list of author information is available at the end of the article chemical bonds or cause electronic transitions in a medium and thereby damage it. As a result, $\mathrm{THz}$ radiation becomes very attractive for various applications related to imaging and diagnostics in areas such as medicine, industrial quality control, food inspection, or artwork examination ${ }^{1-4}$. Moreover, a large number of processes at the microscopic level occur with characteristic times corresponding to terahertz frequencies, for example, rotations and vibrations in large molecules (nucleic acids, proteins, synthetic polymers, etc.), lattice vibrations and free carrier motion in solids. Therefore, $\mathrm{THz}$ waves serve an important role in fundamental studies of matter and find their applications in spectroscopy and the control of materials, including ultrafast electric-field and magneticfield switching, being much faster than in conventional electronics 5 . In addition, there is a whole set of applications that directly rely on strong, high-energy $\mathrm{THz}$ fields, for example, table-top $\mathrm{THz}$ electron acceleration ${ }^{7-9}$ or $\mathrm{THz}$ enhancement of attosecond pulse generation ${ }^{10,11}$.

Despite the existing need for strong $\mathrm{THz}$ radiation, the list of available powerful $\mathrm{THz}$ sources is quite restricted ${ }^{12}$. Currently, there are two major techniques that allow one to generate intense $\mathrm{THz}$ fields on a table top: optical rectification in electro-optic crystals ${ }^{13-15}$ and two-color 


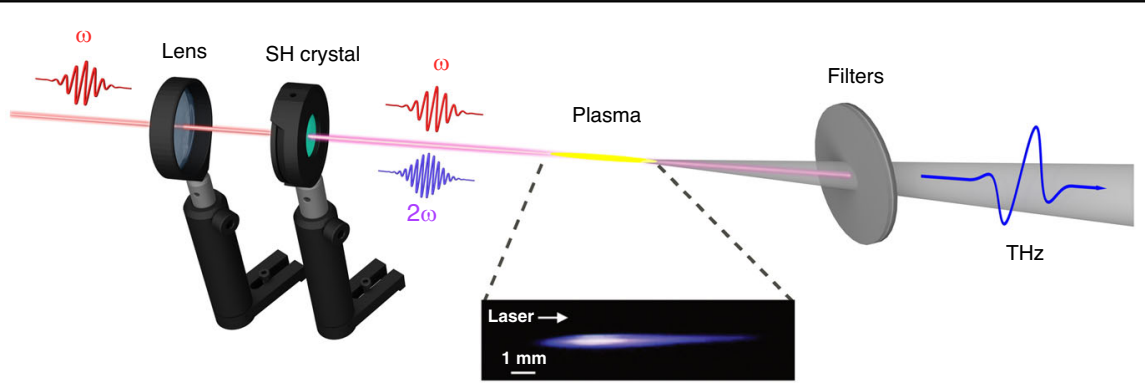

Fig. 1 Typical setup for THz generation by two-color filamentation. The fundamental component of a laser pulse is focused through a nonlinear second harmonic $(\mathrm{SH})$ crystal into ambient air. The second harmonic generated in the nonlinear crystal mixes with the fundamental pulse and provides the desired field symmetry breaking (the phase between the $\omega$ and $2 \omega$ fields is controlled by the crystal position along the optical axis). The plasma channel generated close to the lens focus emits THz radiation, which is then filtered out by a set of longpass filters (in most cases, highresistivity silicon wafers)

filamentation in gases and liquids ${ }^{16-20}$. In THz generation by optical rectification, a crystal with a second-order nonlinearity is pumped by an ultrashort laser pulse; the difference frequency mixing of various spectral components in the wide pulse spectrum produces a beating polarization that gives rise to the emission of radiation in the $\mathrm{THz}$ spectral region. In turn, two-color filamentation is based on the ionization of a medium by an ultrashort laser pulse consisting of fundamental radiation and its second harmonic. Then, free electrons of the generated plasma oscillate in the driving laser field and emit electromagnetic waves. By a proper choice of the phase between the fundamental and second harmonic pulses, it is possible to break the field symmetry and force the free electrons to produce a residual photocurrent oscillating at $\mathrm{THz}$ frequencies ${ }^{21}$.

Among the two above techniques, optical rectification allows one to generate $\mathrm{THz}$ pulses with higher energy, up to $0.9 \mathrm{~mJ}^{14}$, and higher $\mathrm{THz}$ conversion efficiency (the ratio of the $\mathrm{THz}$ energy to the energy of the input twocolor laser pulse), up to $3.7 \%{ }^{22}$. Unfortunately, inevitable optical damage of nonlinear crystals by high laser intensities makes further step ups in $\mathrm{THz}$ energy very troublesome. In addition, $\mathrm{THz}$ pulses generated by optical rectification are spectrally quite narrow (the spectral width is below $5 \mathrm{THz}$ ) and, as a result, are quite long (the corresponding pulse durations are approximately several picoseconds). On the other hand, common two-color filamentation with near-infrared laser pulses offers less energetic $\mathrm{THz}$ radiation (up to $30 \mu \mathrm{J}$ in gases ${ }^{18}$ and up to $80 \mu \mathrm{J}$ in liquids ${ }^{20}$ ) with less $\mathrm{THz}$ conversion efficiency, which is usually close to $0.01 \%$. Nevertheless, since the gas and liquid media recover in-between laser shots, the optical damage issue becomes irrelevant. Moreover, twocolor filamentation allows one to generate ultrashort single-cycle $\mathrm{THz}$ pulses with femtosecond duration and spectral bandwidths exceeding $50 \mathrm{THz}^{23,24}$. Therefore, despite the lower energies, these $\mathrm{THz}$ pulses can have a very high intensity. Furthermore, two-color filamentation makes it possible to generate $\mathrm{THz}$ radiation remotely, thereby avoiding challenges such as the high absorption of $\mathrm{THz}$ waves in atmospheric water vapor ${ }^{25-28}$.

In addition, the $\mathrm{THz}$ radiation generated by optical rectification and two-color filamentation has different emission patterns. Unlike optical rectification, where the spatial profile of the generated $\mathrm{THz}$ radiation repeats the shape of the pump laser beam, the $\mathrm{THz}$ radiation produced by two-color filamentation is emitted as a cone whose angle depends on the thickness of the plasma channel and its length ${ }^{29-31}$.

Figure 1 shows a typical experimental setup for $\mathrm{THz}$ generation by two-color filamentation.

Due to the prevalence of commercially available highpower laser systems operating at near-infrared wavelengths, most experiments on both optical rectification and two-color filamentation were conducted using nearinfrared laser pulses. While the choice of the pump wavelength for optical rectification is mainly dictated by the phase-matching properties of the available nonlinear crystals, the wavelength of the laser source for two-color filamentation can be chosen more freely. Taking into account that many parameters governing the physics of ionization and the motion of electrons in the laser field depend on its wavelength, we can assume that certain laser wavelengths will be beneficial for two-color filamentation. In particular, since the ponderomotive energy of free electrons under the action of a laser field increases as the square of the field's wavelength, it is reasonable to expect that laser sources operating at longer wavelengths should produce stronger photocurrents and, consequently, more energetic $\mathrm{THz}$ radiation. However, to produce filaments with long and dense plasma channels favorable for $\mathrm{THz}$ generation, the peak power of a laser pulse should exceed the critical power for Kerr selffocusing (otherwise, the dense enough plasma can be produced only by tightly focused laser pulses and only in 
the vicinity of the focal spot). In turn, since the critical power for Kerr self-focusing also scales as the wavelength squared, until recently, the experimental investigation of two-color filamentation at wavelengths from mid-infrared and far-infrared parts of the spectrum was almost prohibitive because of the demanding optical power requirement.

Everything changed with the advent of a new generation of optical parametric chirped-pulse amplifiers (OPCPAs) capable of delivering high-peak-power sub-100 fs pulses at a central wavelength of $3.9 \mu \mathrm{m}^{32-34}$. Recent advances in the development of high-power pulsed $\mathrm{CO}_{2}$ lasers are also very encouraging ${ }^{35,36}$. With these newly appeared laser systems, the studies of laser filamentation in gases have been extended to the mid-infrared and far-infrared spectral ranges. The first experiments on longwavelength filamentation revealed a number of unique regimes of nonlinear spatiotemporal wave dynamics and demonstrated many unusual aspects in the nonlinearoptical response of gaseous media to mid-infrared and far-infrared laser radiation ${ }^{37,38}$. One can easily expect that these peculiar laser-matter interactions will also be reflected in the generation of $\mathrm{THz}$ radiation by the twocolor filamentation of long-wavelength laser pulses.

Here, we review the recent experimental and theoretical advances in the interaction of long-wavelength laser radiation with gases in the context of two-color filamentation, which show great promise as sources of extremely powerful $\mathrm{THz}$ waves. We carefully analyze how the complex nonlinear-optical response of gaseous media on mid-infrared and far-infrared radiation can affect the process of $\mathrm{THz}$ generation and what the prerequisites are for stronger and more powerful $\mathrm{THz}$ fields. The theoretical analysis is completed with a review of the current state of research on the nonlinear propagation of midinfrared and far-infrared two-color filaments.

\section{Medium response}

\section{lonization and free electrons}

Ionization plays a central role in the process of $\mathrm{THz}$ generation by two-color laser filamentation. Following the photocurrent model ${ }^{21}, \mathrm{THz}$ radiation originates from the current $J$ of free electrons oscillating under the action of an electric field with broken symmetry. In this framework, the amplitude of the emitted $\mathrm{THz}$ pulses is proportional to the first time derivative of the electron current $\partial J / \partial t$. Since the magnitude of $J$ depends linearly on the electron velocity $v$, the energy of the generated $\mathrm{THz}$ radiation should be proportional to $v^{2}$ and thus to the kinetic energy of the electrons acquired within the electric field.

A classical nonrelativistic equation of motion for an electron driven by a field $E$ can be written as $d v(t) / d t=e E(t) / m$, where $e$ and $m$ are the charge and mass of the electron, respectively. According to this equation, under the action of an electric field $E(t)=A \cos (\omega t)$, the electron gains velocity $v(t)=e A \sin (\omega t) / m \omega$. The corresponding average kinetic energy is

$$
U_{p}=<\frac{m v^{2}}{2}>=\frac{e^{2} A^{2}}{4 m \omega^{2}} \propto I \lambda^{2}
$$

where $A, I$, and $\lambda$ are the field amplitude, intensity, and wavelength, respectively. This energy, known as ponderomotive or quiver energy, is one of the basic parameters governing strong-field physics.

Thus, the most straightforward way to obtain more energetic $\mathrm{THz}$ radiation is to increase the ponderomotive energy $U_{\mathrm{p}}$ of plasma electrons. According to Eq. (1), this can be accomplished either by increasing the pulse intensity $I$ or its wavelength $\lambda$. Obviously, the wavelength is a more effective control knob because $U_{\mathrm{p}}$ depends on $\lambda$ quadratically but only linearly on $I$. Moreover, the maximum laser intensity during filamentation is restricted by the effect of intensity clamping ${ }^{39}$. Since the energy of $\mathrm{THz}$ pulses linearly depends on $U_{\mathrm{p}}$, it should also scale quadratically with the laser wavelength. The latter conclusion is the main motivation for increasing the laser wavelength in studies of $\mathrm{THz}$ sources driven by laser filamentation.

Another important aspect of the ionization process involves the Keldysh parameter $\gamma$, which can be expressed through the ponderomotive energy $U_{\mathrm{p}}$ as

$$
\gamma=\sqrt{\frac{U_{\mathrm{i}}}{2 U_{\mathrm{p}}}} \propto \frac{1}{\sqrt{I} \lambda}
$$

where $U_{\mathrm{i}}$ is the ionization energy of an atom or a molecule. The value of $\gamma$ distinguishes the tunneling $(\gamma \ll 1)$ and multiphoton $(\gamma \gg 1)$ regimes of ionization ${ }^{40}$. For an atom or molecule at constant intensity, $\gamma$ scales as $1 / \lambda$. Therefore, longer wavelengths drive ionization further into the tunneling regime. In turn, an extremely fast, exponential dependence of the tunnel ionization rate on the field amplitude ${ }^{40}$ implies that photoelectrons are released in a narrow range of time close to the peaks of the field. As a result, the maximum ponderomotive energy that an electron can gain will depend on the particular phase of the field at the moment of electron release. Figure 2 shows the temporal evolution of several electron velocities $v(t)$ within the driving laser field for the case of single-color and two-color laser pulses with different phases. In Fig. 2a, one can see that at the end of a singlecolor laser pulse, the final velocities of individual electrons become uniformly distributed around zero, resulting in a zero average photocurrent. The situation is similar in the case of two-color laser pulses with zero phase difference $\phi$ between the fundamental and the second harmonic components (see Fig. 2b). However, when $\phi=\pi / 2$ (see 


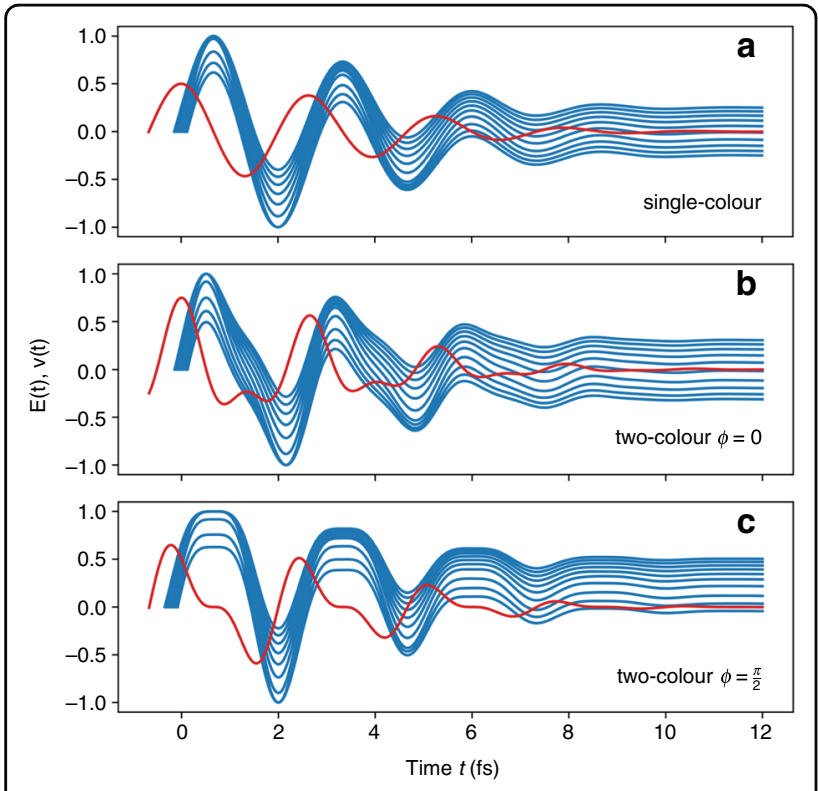

Fig. 2 Mechanism of THz generation by two-color filamentation. Electron velocities $v(t)$ (blue lines) for several electrons released at different time instances close to the peak of the driving field $E(t)$ (red lines). a Single-color laser field $E(t)=A(t) \cos \left(\omega_{0} t\right)$. b , c Two-color laser field $E(t)=A(t) \cos \left(\omega_{0} t\right)+[A(t) / 2] \cos \left(2 \omega_{0} t+\phi\right)$ with $\mathbf{b} \phi=0$ and $\mathbf{c} \phi=\pi / 2$. The amplitude $A(t)=\exp \left(-t^{2} / \tau_{0}^{2}\right)$, where $\tau_{0}=5 \mathrm{fs}$. The central frequency $\omega_{0}$ corresponds to a wavelength of $800 \mathrm{~nm}$

Fig. 2c), most of the electrons at the end of the laser pulse acquire final velocities of the same sign, giving rise to a nonzero residual photocurrent, which is responsible for $\mathrm{THz}$ generation ${ }^{21}$. Thus, the concept of sub-cycle tunnel ionization in a time-varying field becomes central for studies of mid-infrared and far-infrared two-color filamentation. In contrast, in the limit of short wavelengths, corresponding to multiphoton ionization $(\gamma \gg 1)$, the effect of the field phase on the ionization process vanishes. In this case, the ionization probability (measured in inverse seconds) is much less than an optical cycle, and many field periods should pass to release a single free electron. As a result, the ionization rate becomes dependent on the field envelope or, equivalently, on the cycle-averaged intensity rather than the instantaneous field strength. Therefore, the asymmetry introduced by two-color fields smooths out, leading to less efficient $\mathrm{THz}$ generation. However, effective $\mathrm{THz}$ generation in the multiphoton regime can still be achieved if one uses a laser field with two frequencies of noninteger ratio (i.e., $\omega$ and $2 \omega+\delta \omega)$, which result in fast beatings of the pulse envelope $^{41}$.

To date, a large amount of data on the ionization of gases by mid-infrared laser pulses has been obtained in experiments on high-harmonics generation ${ }^{42}$. These experiments confirmed that the kinetic energy of photoelectrons indeed scales as $\lambda^{2}$. Moreover, they

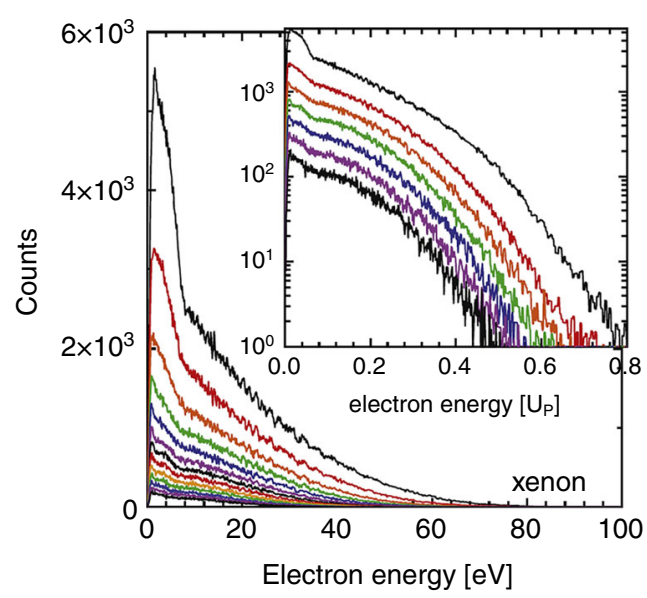

Fig. 3 Low-energy photoelectron spectrum in the tunneling regime. Dependence of the photoelectron spectra of xenon on the intensity of $3.6 \mu \mathrm{m}, 140 \mathrm{fs}$ laser pulses. The laser intensity used to generate the spectra (presented in different colors) was varied in steps of $0.025 I_{0}$, where $I_{0}=0.65 \times 10^{14} \mathrm{~W} \mathrm{~cm}^{-2}$ is the maximum laser intensity. The spectra exhibit a spike-like enhancement for electron energies $\boldsymbol{E} \lesssim 10 \mathrm{eV}$, known as the low-energy structure. The inset displays the spectrum on a semi-logarithmic scale for electron energies in units of ponderomotive energy $U_{p}$. Reprinted from ref. ${ }^{43}$ under the terms of the Creative Commons Attribution 3.0 licence

demonstrated that with an increase in the laser wavelength, the photoelectron energy spectrum evolves towards the structure predicted by the semi-classical theory (known as a simple man model)-a spectrum clipped by $2 U_{\mathrm{p}}$ followed by a plateau descending to the $10 U_{\mathrm{p}}$ cutoff caused by photoelectron rescattering and reacceleration by the field ${ }^{43}$. Thus, the physics of ionization and motion of photoelectrons with increasing laser wavelength tends closer to the classical scenario.

Apart from the confirmation of theoretical expectations, experiments on high harmonic generation with midinfrared laser pulses led to the discovery of a previously unknown feature in the energy distribution of photoelectrons. It was found that the lowest energy part of the photoelectron energy spectrum has an unexpected peak, the so-called low-energy structure, which becomes prominent only when using long-wavelength laser pulses ${ }^{43-45}$ (see Fig. 3). Interestingly, it appears that these low-energy electrons are tightly connected to the emitted $\mathrm{THz}$ waves and can shed light on the microscopic mechanisms of $\mathrm{THz}$ generation ${ }^{46}$.

Another distinguishable feature of ionization driven by long-wavelength laser pulses is related to the increasing role of free electrons in the overall nonlinear response of a medium. The solution of the Schrödinger equation for a generic hydrogen quantum system shows that for highintensity long-wavelength fields, electrons released after ionization can travel far away from the atomic core, acquiring high energy within the long field period ${ }^{47}$. In 

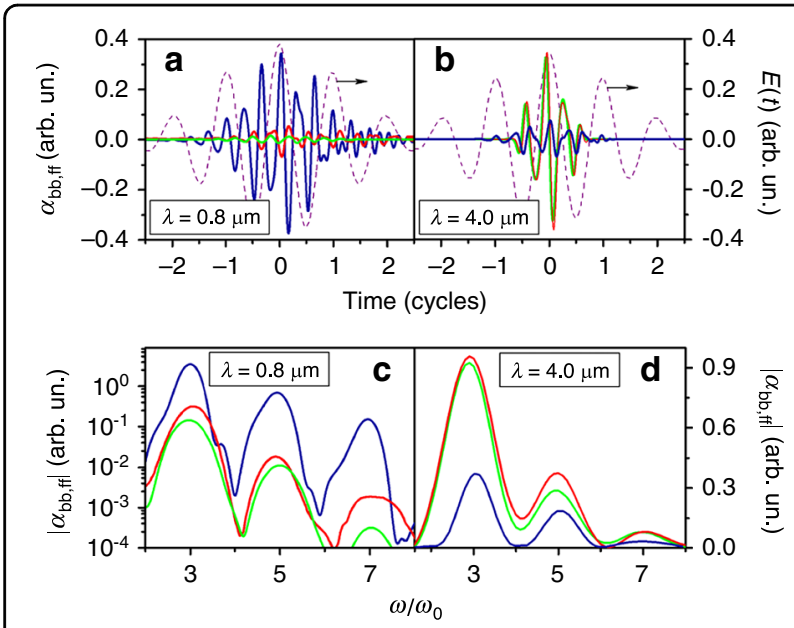

Fig. 4 Comparison of radiation amplitudes of bound and free electrons at different laser wavelengths. Time-resolved radiation amplitudes $a_{\mathrm{bb}}$ (blue) and $a_{\mathrm{ff}}$ (red) of bound-state and free electrons $\mathbf{a}, \mathbf{b}$ and their spectra $\mathbf{c}, \mathbf{d}$ for a laser pulse (purple dashed line) with an intensity of $110 \mathrm{TW} / \mathrm{cm}^{2}$ and wavelengths of $0.8 \mu \mathrm{m} \mathbf{a}, \mathbf{c}$ and $4 \mu \mathrm{m}$ $\mathbf{b}$, $\mathbf{d}$. The results of calculations using the semi-classical model for free electrons are indicated by the green line. Reprinted from ref. ${ }^{47}$ with permission from the American Physical Society

this regime, the electron wave function is no longer tightly localized around the atomic core, and a significant fraction of electrons undergoing ionization do not recombine back to bound states, building up the continuum population in a stepwise fashion after each field half-cycle. The steps in the continuum population, synchronized with the field half-cycle, translate into harmonics whose intensity is much higher than the intensity of harmonics emitted by bound-state electrons (see Fig. 4). As a result, for highintensity long-wavelength pulses, free-state electrons dominate over bound electrons in the overall nonlinear response. Thus, since for longer laser wavelengths, the behavior of free electrons tends to be more classical, the use of simple semi-classical models for studies of midinfrared and far-infrared two-color filamentation seems appropriate.

To complete the semi-classical description of ionization for long-wavelength laser pulses, we have to indicate the way to calculate the corresponding ionization rate. Recently, Lai et al. ${ }^{48}$ experimentally studied the ionization yield versus laser intensity for various atomic targets irradiated by laser pulses with different polarizations and wavelengths ranging from the visible to the mid-infrared $(0.4-4 \mu \mathrm{m})$ in both the multiphoton and tunneling regimes. The experimental findings were compared to the ionization rate calculated by the Perelomov-Popov-Terent'ev (PPT) formula ${ }^{49}$. It was shown that the PPT formula, corrected by a generalized Coulomb prefactor ${ }^{50}$, agrees well with all of the experimental data, including the ionization yield ratio between circular and linear laser polarizations.

The estimations of classical electron trajectories under the action of strong mid-infrared and far-infrared laser fields show that tunneling electrons can travel away from the parent atom or molecule over distances comparable to a collisional mean free path in gases ${ }^{38}$. Consequently, an electron released from a parent atom or molecule can interact with many other atoms or molecules located nearby. In particular, on the way from its birthplace, such an electron can acquire enough energy to ionize neighboring atoms or molecules and give rise to significant avalanche ionization. Thus, compared to near-infrared laser fields, the theoretical description of laser-plasma interactions with mid-infrared and far-infrared laser pulses requires paying more attention to many-body effects.

For example, recent theoretical studies suggest that many-body effects in gases can lead to an additional ionization channel through excitation-induced dephasing (EID), which manifests itself at low laser intensities ${ }^{51-54}$. It was found that the following semi-phenomenological formula for the ionization rate provides a good fit to the full many-body quantum theory of $\operatorname{EID}^{38,54}$ :

$$
R(E)=C_{\mathrm{MBI}} E^{4}(t) \sqrt{\frac{E^{2}(t)+s}{E^{2}(t)}}
$$

with $C_{\mathrm{MBI}}=2.84 \times 10^{-7}\left(\mathrm{~m}^{-3} \mathrm{~s}^{-1}\right)\left(\mathrm{m}^{4} \mathrm{~V}^{-4}\right)$ and $s=4.6 \times$ $10^{18} \mathrm{~V}^{2} \mathrm{~m}^{-2}$. According to Eq. (3), the additional ionization yield proposed by EID calculations scales quadratically with the laser intensity $I$, in strong contrast to the much higher intensity powers predicted by the multiphoton ionization model. The validity of EID calculations was tested by comparing the results of comprehensive propagation simulations with data from experiments on the filamentation of terawatt picosecond $\mathrm{CO}_{2}$ laser pulses ${ }^{55}$. It was shown that the plasma electrons produced through the EID mechanism provide a necessary seed for avalanche ionization, which then produces enough electrons to stabilize the laser pulse propagation, leading to extremely long and wide filaments.

The apparent discrepancy between the experiments of Lai et al. ${ }^{48}$, showing the validity of the PPT ionization rate formula, and the EID calculations can be explained by the fact that the experiments by Lai et al. were conducted at low gas pressures, where the many-body interactions lose their importance. To clarify this situation, Woodbury et al. ${ }^{56}$ experimentally measured the ionization yield in air, nitrogen, and argon at atmospheric (0.5-3 bar) pressures for 1.024 and $3.9 \mu \mathrm{m}$ picosecond laser pulses. The ionization yield was measured over a dynamic range of intensities covering 14 orders of magnitude (the sensitivity of the experiment allowed to observe individual ionization events at low intensities). The obtained 
experimental results can be summarized as follows. At low intensities $\left(<4 \mathrm{TW} / \mathrm{cm}^{2}\right)$, where the largest relative contribution from EID ionization is expected, the ionization yield was consistent with the multiphoton ionization rate of an unknown contaminant (found in all considered gases) with ionization energy $U_{\mathrm{i}}=6 \mathrm{eV}$. Neither the wavelength-independent $I^{2}$ scaling nor the wavelength-insensitive absolute yield suggested by EID was observed. In addition, the measured yields were a factor of $10^{6}$ lower than those predicted by EID. At higher intensities $\left(>10 \mathrm{TW} / \mathrm{cm}^{2}\right)$, the ionization yields were consistent with the multiphoton or tunneling ionization rates of isolated molecules calculated by the PPT formula $^{49}$ with a generalized Coulomb prefactor ${ }^{50}$. At the moment, the source of the aforementioned contradiction between the experiments and theory remains unclear and requires further study.

Recent numerical simulations showed that seed electrons for avalanche ionization can come from the ionization of air aerosols ${ }^{57}$. Similar to the EID mechanism, this scenario can explain the appearance of additional free electrons (which otherwise cannot be produced through an avalanche seeded solely by tunnel ionization), which are necessary to obtain the long and stable far-infrared filaments observed in the experiment $t^{55}$.

In addition to the ionization yield measurements, Woodbury et al. ${ }^{56}$ estimated the effective collisional frequency $v_{\mathrm{c}}$, which determines the avalanche ionization rate. $v_{\mathrm{c}}=0.55 \mathrm{ps}^{-1}$ was found for both the 1.024 and $3.9 \mu \mathrm{m}$ wavelengths. However, in view of the growing role of many-body effects in laser-plasma interactions with mid-infrared and far-infrared laser pulses, a recent theoretical study by Wright et al. $^{58}$ put the simple, oneparameter description of the avalanche ionization rate into question. They proposed a two-temperature model, which provides a microscopically motivated foundation for avalanche ionization in gases with long-wavelength laser pulses. The basic idea behind this model is that free electrons, moving in the laser field after photoionization occurs, need some time to acquire a large enough kinetic energy to collisionally ionize neighboring atoms or molecules. As a consequence, avalanche ionization does not instantaneously follow the laser intensity, leading to memory or transient effects. In particular, the plasma density can continue to increase well after the pulse has passed due to the presence of high-energy electrons that store enough energy for impact ionization of the neutral atoms or molecules at later times.

The increasing role of free electrons in the overall nonlinear response of a medium can lead to even more exotic scenarios. For example, numerical simulations by Gao and Shim ${ }^{59}$ showed that a $15 \mu \mathrm{m}$ laser pulse propagating in a weakly ionized argon gas can undergo selffocusing (at a power much lower than the critical power for Kerr self-focusing) due to the transverse variations in intensity-dependent electron-impact ionization.

From the above discussion, it becomes clear that one should be very careful in modeling the ionization and electron dynamics driven by mid-infrared and far-infrared fields. Electron densities and energy distributions sensitively depend on the driving field wavelength, and there are still a number of blind spots and controversies in our understanding of these dependences. However, the classical treatment of electron dynamics allowed at these frequencies can greatly simplify the theory. To date, the simulations of $\mathrm{THz}$ generation from mid-infrared filamentation account for the simplified theory and seem to reproduce the experimental findings well, as we will show in the following.

\section{Nonlinear polarization: Kerr and Raman effects}

The effect of self-focusing lies in the basis of laser filamentation and, alongside ionization, is responsible for the formation of the extended plasma channels necessary for effective $\mathrm{THz}$ generation. This nonlinear effect leads to an intensity-dependent change in the total refractive index $n=n_{0}+n_{2} I$, where $n_{0}$ is the linear part of the refractive index, $n_{2}$ is the nonlinear index, and $I$ is the laser intensity. In molecular gases, such as nitrogen or oxygen, there are two contributions to the nonlinear index $n_{2}$. The first one (Kerr) is nearly instantaneous and originates from the nonlinear response of bound electrons to the external electric field. The second one (Raman) is time-dependent and arises from the tendency of gas molecules to align themselves along the field polarization. Due to inertia during the rotation of molecules, the molecular alignment lags behind the driving field and results in a delayed increase in polarizability, which, in turn, leads to an increase in the refractive index near the trailing end of the laser pulse. The combination of these two effects gives rise to a nonlinear polarization, $P_{\mathrm{nl}}$, which can be modeled as

$$
P_{\mathrm{nl}}(r, t)=\varepsilon_{0} X^{(3)}\left[\left(1-f_{\mathrm{R}}\right) E^{2}(r, t)+f_{\mathrm{R}} \int_{-\infty}^{t} R\left(t-t^{\prime}\right) E^{2}\left(r, t^{\prime}\right) d t^{\prime}\right] E(r, t)
$$

Here, $X^{(3)}$ is the total (Kerr plus Raman) third-order susceptibility, and $f_{\mathrm{R}} \in[0,1]$ is the fraction of the Raman contribution. For subpicosecond laser pulses, the response function $R(t)$ can be calculated based on the damped harmonic oscillator model ${ }^{60,61}$ :

$$
R(t)=R_{0} \exp (-\Gamma t / 2) \sin (\Lambda t)
$$

where $R_{0}=\left(\Gamma^{2} / 4+\Lambda^{2}\right) \Lambda^{-1}$ is a normalization factor and $\Gamma$ and $\Lambda$ are the damping and oscillation frequencies, respectively. 
The total nonlinear index $n_{2}$ linearly depends on $\chi^{(3)}$ and can be written as $n_{2}=3 X^{(3)}\left(4 \varepsilon_{0} n_{0}^{2} c_{0}\right)^{-1}$. We can express $n_{2}$ as a sum of nonlinear indices $n_{2 \mathrm{~K}}$ and $n_{2 \mathrm{R}}$, responsible for, respectively, the Kerr and Raman contributions: $n_{2}=n_{2 \mathrm{~K}}+n_{2 \mathrm{R}}$, where $n_{2 \mathrm{~K}}=\left(1-f_{\mathrm{R}}\right) n_{2}$ and $n_{2 \mathrm{R}}=f_{\mathrm{R}} n_{2}$.

Since most of the experiments on two-color filamentation, due to their simplicity, are conducted in air, let us consider the nonlinear indices of air in more detail. While the values of the Kerr nonlinear index $n_{2 K}$ for air have been measured for various wavelengths, from the ultraviolet up to the near-infrared parts of the spectrum, until recently, there were no corresponding data for the midinfrared and far-infrared spectral range. Currently, the most comprehensive measurements of $n_{2 \mathrm{~K}}$ for different air constituents were made by Zahedpour et al. ${ }^{62,63}$ using the single-shot supercontinuum spectral interferometry technique. They presented the experimental values of the Kerr nonlinear index for nitrogen and oxygen in a very wide range of wavelengths from 0.4 to $11 \mu \mathrm{m}$. With these data, we can calculate the values of $n_{2 \mathrm{~K}}$ for air as a weighted sum $n_{2 \mathrm{~K}}=0.79 n_{2 \mathrm{~K}}^{\left(N_{2}\right)}+0.21 n_{2 \mathrm{~K}}^{\left(O_{2}\right)}$, where $n_{2 \mathrm{~K}}^{\left(N_{2}\right)}$ and $n_{2 \mathrm{~K}}^{\left(\mathrm{O}_{2}\right)}$ are the Kerr nonlinear indices of nitrogen and oxygen, while the factors 0.79 and 0.21 are the typical fractions of these gases in air. Figure $5 \mathrm{a}$ shows the resulting wavelength dependence of the Kerr nonlinear index $n_{2 K}$ for air. Evidently, despite some noise, the obtained values of $n_{2 \mathrm{~K}}$ demonstrate that over the studied range of wavelengths, the Kerr nonlinear response of air is quite dispersionless.

In turn, Brown et al. ${ }^{64}$ used ab initio calculations based on the time-dependent density functional theory to compute the Kerr nonlinear index $n_{2 \mathrm{~K}}$ for nitrogen and oxygen at near-infrared and mid-infrared wavelengths in the range from 1 to $4 \mu \mathrm{m}$. It was shown that the calculated values of $n_{2 \mathrm{~K}}$ can be well fitted by a Sellmeier-like equation:

$$
n_{2 \mathrm{~K}}(\lambda)=\frac{P^{-1}}{\lambda_{0}^{-2}-\lambda^{-2}}
$$

where $P=14.63 \mathrm{GW}, \lambda_{0}=0.3334 \mu \mathrm{m}$ for nitrogen and $P=14.62 \mathrm{GW}, \lambda_{0}=0.3360 \mu \mathrm{m}$ for oxygen, with the wavelength $\lambda$ measured in $\mu \mathrm{m}$ (as before, the nonlinear index for air can be calculated as the corresponding weighted sum). Figure 5a shows that Eq. (6) provides a very good fit for the experimental data obtained by Zahedpour et al. ${ }^{62,63}$ and confirms the flat and featureless landscape of air $n_{2 \mathrm{~K}}$ dispersion at mid-infrared and farinfrared wavelengths.

Additionally, Brown et al. ${ }^{64}$ found that over the wavelength range of $2-4 \mu \mathrm{m}$, the Kerr nonlinear index $n_{2 K}$ of air is insensitive to the laser intensity up to intensities as high as $10^{14} \mathrm{~W} / \mathrm{cm}^{2}$ (though at shorter wavelengths, a weak intensity dependence, caused by nonnegligible
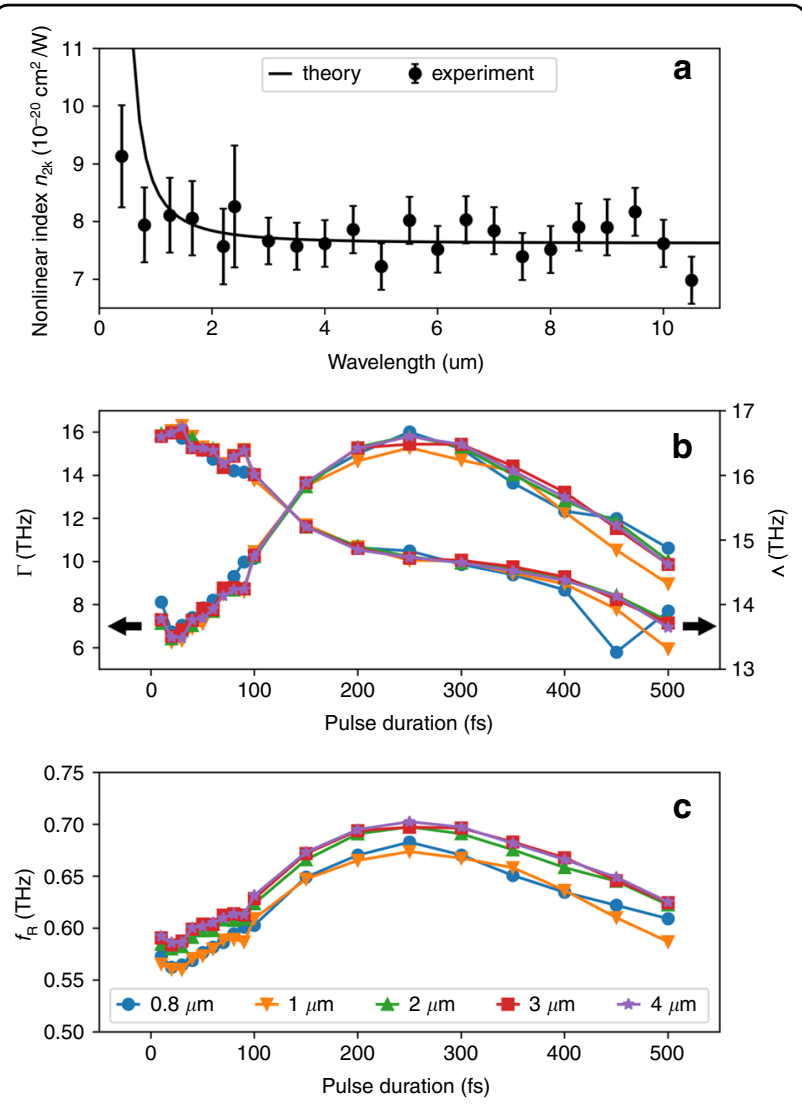

Fig. 5 Parameters of Kerr and Raman contributions to the nonlinear index of air. a Dependence of the Kerr nonlinear index for air on the laser wavelength: experimental data obtained by Zahedpour et al. ${ }^{62,63}$ (points) and theory developed by Brown et al. ${ }^{64}$ according to Eq. (6) (line). $\mathbf{b}$ Values of frequencies $\Gamma$ and $\wedge$ determining the Raman response function $R(t)$ (arrows indicate the associated scale), and $\mathbf{c}$ the fraction of the Raman contribution $f_{R}$ in air as a function of the pulse duration for wavelengths of $0.8-4 \mu \mathrm{m}$. Figures $\mathbf{b}$ and $\mathbf{c}$ are reprinted from ${ }^{61}$ with permission from the American Physical Society

ionization, was observed for intensities above $5 \times 10^{13} \mathrm{~W} /$ $\mathrm{cm}^{2}$ ). These findings are in line with recent experiments that showed that for near-infrared laser pulses, the nonlinear response of bound electrons preserves its linear dependence on intensity even in the presence of substantial ionization, well beyond the limits of perturbation theory ${ }^{65}$. In turn, any dependence of the Kerr nonlinear index $n_{2 \mathrm{~K}}$ on intensity would signal that there are some nonlinearities of higher order that we do not take into account. Since with increasing wavelength, ionization tends to occur in the tunnel regime and thus becomes independent of the wavelength, one can assume that the above conclusions on the independence of $n_{2 \mathrm{~K}}$ from the intensity can be safely extended to laser pulses with even longer wavelengths located further in the far-infrared spectral region. Thus, we can conclude that in the range of wavelengths from near-infrared to far-infrared and at 
intensities up to $10^{14} \mathrm{~W} / \mathrm{cm}^{2}$, the dominant contribution to the nonlinear polarization comes from the effects of the third order, while higher orders of nonlinearity can be neglected.

In addition to the $n_{2 K}$ values, Zahedpour et al. ${ }^{62,63}$ also measured the Raman nonlinear index $n_{2 \mathrm{R}}$ for nitrogen and oxygen in the same wavelength range from 0.4 to $11 \mu \mathrm{m}$. According to the obtained results, the value of $n_{2 R}$ for air (calculated through the corresponding weighted sum) is constant over the considered wavelength range and equal to approximately $3 \times 10^{-20} \mathrm{~cm}^{2} / \mathrm{W}$. Taking into account the values of the Kerr nonlinear index $n_{2 \mathrm{~K}}$ presented in Fig. $5 \mathrm{a}$, this $n_{2 R}$ value suggests a fraction of the Raman contribution $f_{\mathrm{R}}=n_{2 \mathrm{R}} /\left(n_{2 \mathrm{~K}}+n_{2 \mathrm{R}}\right) \approx 0.8$.

In parallel, Langevin et al. ${ }^{61}$ numerically studied the molecular Raman contribution in air at mid-infrared wavelengths. By solving the time-dependent Schrödinger equation, they calculated the rotational molecular response of air for laser pulses with wavelengths from 0.8 to $4 \mu \mathrm{m}$, pulse durations from 10 to $500 \mathrm{fs}$, and intensities from 0.2 to $20 \mathrm{TW} / \mathrm{cm}$. It was demonstrated that the treatment of the Raman contribution by Eq. (4) with the damped harmonic oscillator response function given by Eq. (5) can be safely extended to mid-infrared wavelengths. Figure $5 \mathrm{~b}$ shows the two frequencies, $\Gamma$ and $\Lambda$, calculated by Langevin et al. $^{61}$ for air in the range of different wavelengths and pulse durations. According to this figure, the values of $\Gamma$ and $\Lambda$ are almost independent of the wavelength but exhibit a moderate dependence on the pulse duration. In turn, Fig. $5 \mathrm{c}$ shows that, depending on the pulse duration, the value of Raman fraction $f_{R}$ smoothly changes in the range from 0.55 to 0.7 but, similar to the characteristic frequencies, remains nearly independent of the wavelength.

From Eq. (5), we find that the peak of the response function $R(t)$ occurs at time $t_{\mathrm{p}}=\Lambda^{-1} \tan ^{-1}(2 \Lambda / \Gamma)$. According to the values of $\Gamma$ and $\Lambda$ calculated by Langevin et al. ${ }^{61}, t_{\mathrm{p}}$ lies in the range between 75 and $90 \mathrm{fs}$, which roughly indicates the pulse durations above which the Raman contribution will have a significant impact on the refractive index change. Therefore, taking into account the above values of $f_{\mathrm{R}}$, we can conclude that for laser pulses with durations above approximately $75 \mathrm{fs}$, the molecular Raman contribution prevails over the electronic Kerr response for all wavelengths from the nearinfrared to the mid-infrared region.

In addition, numerical simulations of laser filamentation in air performed by Langevin et al. ${ }^{61}$ demonstrated that the Raman contribution manifests itself in exactly the same way (through a considerable red-shift of the laser spectrum) for all near-infrared and mid-infrared laser pulses.

We also mention the experiments by Pigeon et al. ${ }^{66,67}$, where the total nonlinear index of air $n_{2}=50 \times 10^{-20}$ $\mathrm{cm}^{2} / \mathrm{W}$ was measured for $200 \mathrm{ps}, 10.6 \mu \mathrm{m}$ laser pulses generated by a pulsed $\mathrm{CO}_{2}$ laser. However, due to the long pulse duration and the time-integrated measurements, the Kerr and Raman contributions in these experiments cannot be separated.

In summary, the above results show that the nonlinear polarization response of air at mid-infrared and farinfrared wavelengths is explored in depth, both experimentally and theoretically. The good quantitative agreement between the theory and experimental data indicates that the basic underlying physics is well understood, while the obtained values of the Kerr and Raman response parameters can be used for comprehensive simulations of $\mathrm{THz}$ generation by mid-infrared and far-infrared twocolor filamentation.

\section{Dispersion of air refractive index}

Due to its simplicity, most of the experiments on $\mathrm{THz}$ generation by two-color filamentation are conducted in air. By itself, air is a mixture of different gases, including nitrogen $\mathrm{N}_{2}$, oxygen $\mathrm{O}_{2}$, water vapor $\mathrm{H}_{2} \mathrm{O}$, carbon dioxide $\mathrm{CO}_{2}$, and several others. Depending on its wavelength, electromagnetic radiation propagating in air can fall in resonance with various electronic transitions, as well as vibrations and rotations in the above molecular gases, which will result in the absorption of this radiation. As a result, only radiation of specific wavelengths, lying in socalled transparency windows, can propagate in air without losses. Therefore, before developing any specific laser system for two-color filamentation in air, it is necessary to ensure that the wavelengths of its fundamental and second harmonic fall into one of these transparency windows. This is especially important for laser pulses from mid-infrared and far-infrared parts of the spectrum, which are densely populated by absorption lines corresponding to the vibrations and rotations of $\mathrm{H}_{2} \mathrm{O}$ and $\mathrm{CO}_{2}$ molecules.

Figure 6a shows the real part of the air refractive index $n(w)$, as well as the corresponding absorption coefficient $\alpha(w)$ calculated using the HITRAN database ${ }^{68,69}$ for wavelengths in the range from 0.5 to $12 \mu \mathrm{m}$. We can see that the presence of $\mathrm{H}_{2} \mathrm{O}$ molecules in air gives rise to several groups of absorption lines between 1.3 and $3 \mu \mathrm{m}$, as well as to a wide absorption band located between 5 and $7.5 \mu \mathrm{m}$. In turn, the presence of $\mathrm{CO}_{2}$ molecules leads to the strong absorption of radiation at wavelengths from 4.1 to $4.4 \mu \mathrm{m}$. With this knowledge of air absorption bands, we can determine the wavelengths of possible laser sources suitable for two-color filamentation. The shaded areas in Fig. 6 indicate the spectral regions where the central wavelength of either the fundamental pulse or its second harmonic falls into one of the absorption bands. As we can see, the choice of allowed wavelengths in the mid-infrared and far-infrared part of the spectrum is quite restricted. 

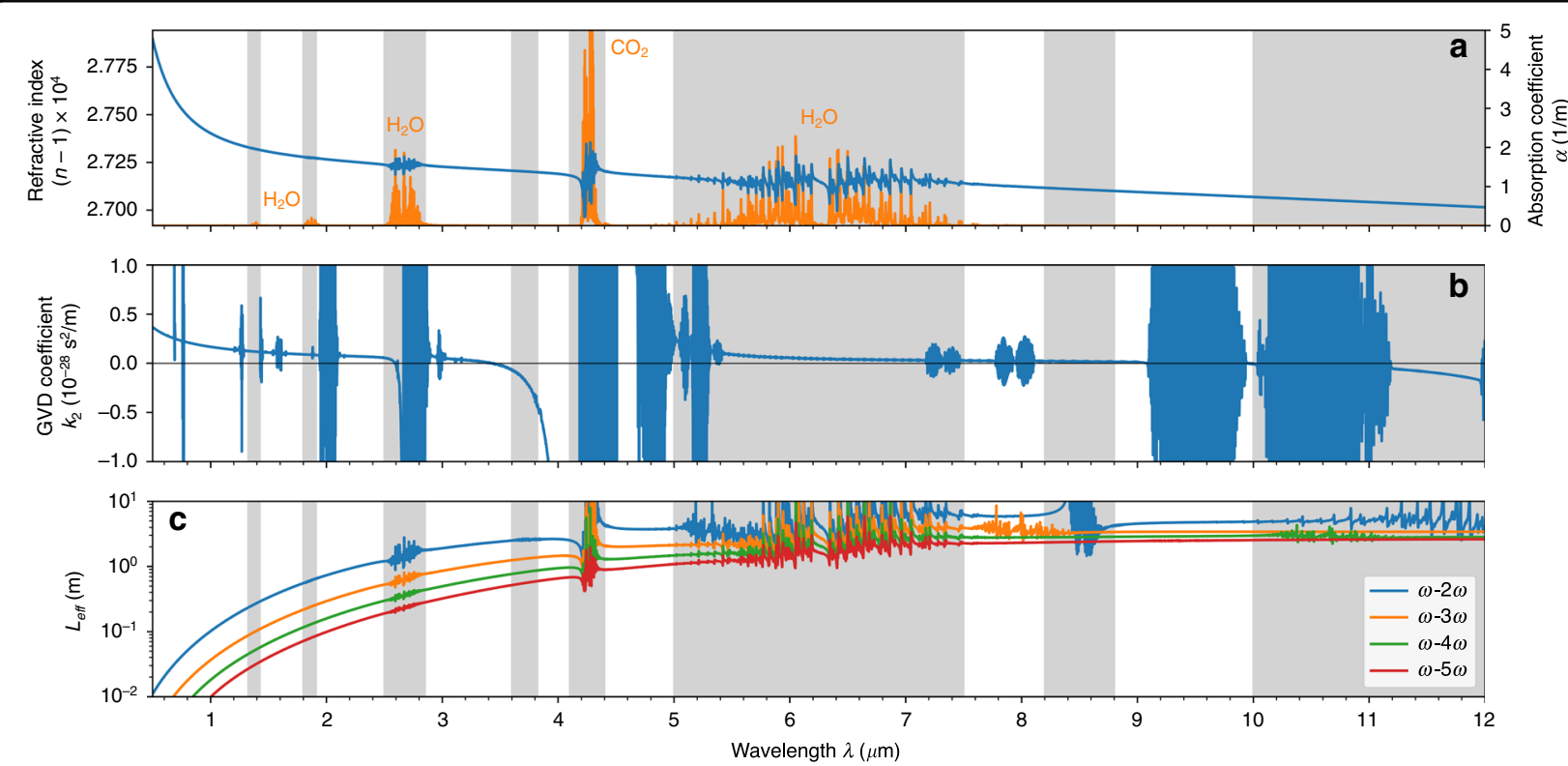

Fig. 6 Dispersion of air. Wavelength dependence of a the refractive index $n$ (blue) and absorption coefficient $a$ (orange), $\mathbf{b}$ the group velocity dispersion coefficient $k_{2}$, and (c) the phase velocity walk-off length $L_{\text {eff }}$ for several pairs of frequencies

One should also be aware of another phenomenon that can potentially shrink the selection of possible wavelengths even further. Filamentation of ultrashort laser pulses is accompanied by broadening of their spectra and the generation of a supercontinuum. Therefore, if the central wavelength of a laser pulse is located close to one of the absorption bands, a part of the nonlinearly expanding spectrum will penetrate the region of resonance frequencies and will be continuously absorbed ${ }^{69,70}$. Under certain conditions and especially under loose focusing, this nonlinearly enhanced, through spectral broadening, linear absorption can dominate other sources of losses and strongly alter the process of filamentation and generation of plasma ${ }^{69}$.

Among all of the air transparency windows, the one from 3.8 to $4.1 \mu \mathrm{m}$ draws special attention. As shown in Fig. $6 \mathrm{~b}$, this spectral range is characterized by large negative values of the group velocity dispersion coefficient $k_{2}=\partial^{2} k / \partial \omega^{2}$, which corresponds to anomalous group velocity dispersion. The propagation of ultrashort laser pulses in anomalously dispersive nonlinear media is characterized by temporal self-compression and the formation of quasi-solitonic light bullets that lead to the formation of more uniform, elongated plasma channels, thus providing more favorable conditions for $\mathrm{THz}$ generation.

One of the essential prerequisites for efficient $\mathrm{THz}$ generation by two-color filamentation is the properly chosen phase difference between the fundamental and second harmonic pulses, which guarantees optimal field symmetry breaking and thus maximizes the photocurrent oscillating at THz frequencies (see Fig. 2). However, since the air refractive index varies with frequency, the fundamental and second harmonic waves propagate at different phase velocities. As a result, the optimal $\mathrm{THz}$ generation phase difference can be sustained over only a limited propagation length. We can estimate this length as $L_{\text {eff }}=(\lambda / 2) /|n(\omega)-n(2 \omega)|$, where $\lambda=2 \pi c_{0} / \omega$ is the central wavelength of the fundamental wave and $n(\omega)$ and $n(2 \omega)$ are the linear refractive indices at a given frequency. The effective length $L_{\text {eff }}$ corresponds to the distance where the phase difference between the fundamental and second harmonic waves changes by $\pi$ and indicates the maximum length of effective $\mathrm{THz}$ generation. Figure $6 \mathrm{c}$ shows the dependence of $L_{\text {eff }}$ on the wavelength $\lambda$. It is clearly seen that during the transition from the nearinfrared to the mid-infrared spectral range, the effective length $L_{\text {eff }}$ rapidly increases until it saturates in the far infrared region. For comparison, for laser wavelengths of $0.8,3.9$, and $10 \mu \mathrm{m}, L_{\text {eff }}$ is equal to $5 \mathrm{~cm}, 2.6 \mathrm{~m}$, and $4.9 \mathrm{~m}$, respectively. Thus, by using laser pulses with longer wavelengths, it is possible to maintain the optimal phase difference between the fundamental and second harmonic pulses over a longer propagation distance, thereby increasing the length of efficient $\mathrm{THz}$ generation.

Beyond the phase difference discussed above and its implications, the difference in group velocities of the fundamental and second harmonic pulses leads to a temporal walk-off between the two-color pulses along the propagation. This temporal walk-off for a given distance $z$ can be calculated as: $\Delta t_{g}=\left[v_{g}^{-1}(w)-v_{g}^{-1}(2 w)\right] z$, where $v_{g}(w)$ and $v_{g}(2 w)$ are the group velocities of the 
fundamental wave and its second harmonic, respectively. For example, after one meter of propagation in air of fundamental pulses with central wavelengths of $0.8,3.9$, and $10 \mu \mathrm{m}$, their second harmonics will lag behind by, respectively, $81,0.15$, and $2.2 \mathrm{fs}$. Therefore, we find that the group velocity walk-off reduces in the mid-infrared and far-infrared spectral regions, thereby making preferable the choice of longer driving wavelengths for twocolor filamentation.

Apart from the effective length $L_{\text {eff }}$ the waves at fundamental and second harmonic frequencies $(\omega-2 \omega)$, Fig. 6c also shows $L_{\text {eff }}$ for several other harmonics $(\omega-3 \omega, \omega-4 \omega$, and $\omega-5 \omega)$ appearing during two-color filamentation as a part of the generated supercontinuum. Similar to the case of the second harmonic, the corresponding values of $L_{\text {eff }}$ increase with wavelength. In other words, the higher harmonics of long-wavelength laser pulses remain in phase with the wave at the fundamental frequency over a considerable propagation distance. As a result of this continuous phase locking, the underlying optical carrier wave exhibits extreme shock formation, as in the case of an ideal nondispersive medium ${ }^{71}$. Under certain conditions, in the case of collimated or weakly focused laser beams, this shock wave formation may occur well before ionization can generate a sufficient amount of plasma to stop the intensity growth caused by Kerr self-focusing ${ }^{72}$. In this situation, shock wave formation becomes a dominant mechanism of intensity clamping, which may no longer require the generation of plasma. Under these loose focusing conditions and in the absence of a sufficient number of free electrons, one can expect the suppression of $\mathrm{THz}$ generation.

A recent theoretical study predicts another unusual nonlinear-optical effect caused by anomalous dispersion of the air refractive index ${ }^{73}$. It was shown that nonlinear propagation of a long-wavelength ultrashort laser pulse in a medium with a broad and weak region of anomalous dispersion (as an example, the authors considered $100 \mathrm{fs}$ pulses at a $10 \mu \mathrm{m}$ wavelength) can be accompanied by the formation of attosecond $(\approx 0.66 \mathrm{fs})$ subcycle ripples on the temporal pulse profile. It was predicted that these ripples can occur during the initial stage of propagation, well before the self-focusing collapse point. The above results echo earlier theoretical studies that predicted the formation of intense attosecond shock waves for near-infrared laser pulses $^{74}$. In general, the appearance of extremely short subcycle formations with high intensity can locally enhance the yield of tunneling electrons and thus alter their acceleration and deceleration phases in a laser field. As a result, one can expect a strong disturbance of the optimal phase between the fundamental and second harmonic waves and thereby disruption of the process of $\mathrm{THz}$ generation.

In summary, the linear medium absorption places strict limits on the operational spectral windows where nonlinear propagation can be effectively studied, while anomalous dispersion regions can be useful for solitoniclike propagation.

In the following, we use the microscopic description of light-matter interactions with intense mid-infrared and far-infrared light fields, discussed in detail in the last three sections, to explore the generation of $\mathrm{THz}$ radiation from mid-infrared and far-infrared filaments.

\section{THz generation}

Early evidence of stronger $\mathrm{THz}$ radiation at midinfrared wavelengths was obtained from particle-in-cell (PIC) simulations of $4 \mu \mathrm{m}$ single-color laser pulses interacting with a hydrogen gas target ${ }^{75}$. It was shown that the amplitude of the emitted $\mathrm{THz}$ pulses is enhanced by 35 times as the laser wavelength increases from 1 to $4 \mu \mathrm{m}$. However, since the single-color pulses cannot provide effective field symmetry breaking and PIC simulations do not take into account propagation effects, it was difficult to extrapolate these results to the case of actual experiments on two-color filamentation.

In a later study, a comprehensive model based on the unidirectional pulse propagation equation (UPPE) was used to simulate $\mathrm{THz}$ generation by two-color laser filamentation in $\operatorname{argon}^{76}$. Among other things, the authors compared two-color filamentation with 0.8 and $2 \mu \mathrm{m}$ laser pulses and showed that, in the latter case, the $\mathrm{THz}$ energy is approximately ten times higher.

The first experimental study of $\mathrm{THz}$ generation by twocolor filamentation at different wavelengths, in the range from 0.8 to $2.02 \mu \mathrm{m}$, was carried out by Clerici et al. ${ }^{77}$. The obtained results, reproduced in Fig. 7a, clearly show a fast increase in the $\mathrm{THz}$ energy with increasing laser wavelength $\lambda$. The growth of the THz yield extended up to a wavelength of $1.85 \mu \mathrm{m}$, where the $\mathrm{THz}$ energy reached its maximum value of approximately $0.65 \mu \mathrm{J}$, which is approximately 30 times larger than that at $0.8 \mu \mathrm{m}$. The strength of the corresponding $\mathrm{THz}$ field was estimated to be $4.4 \mathrm{MV} / \mathrm{cm}$. However, at wavelengths above $1.85 \mu \mathrm{m}$, the observed $\mathrm{THz}$ energy unexpectedly decreased. In addition, the initial increase in the $\mathrm{THz}$ yield could be well fitted by a $\lambda^{4.6}$ power law, while the photocurrent model predicts at most a $\lambda^{2}$ dependence. The authors partially justified the observed deviations from the theory by their specific experimental conditions, such as the focusing and input energy limitations. Another suggested idea was that the length and radius of the generated plasma channels depend on the laser wavelength, which introduces a correction to the $\lambda^{2}$ scaling and could explain the saturation of THz energy at longer wavelengths.

Subsequent theoretical studies questioned the very possibility of describing the wavelength-dependent scaling of the $\mathrm{THz}$ yield as a simple $\lambda^{a}$ power law with a universal power $\alpha^{79}$. For the example of two-color laser pulses with 

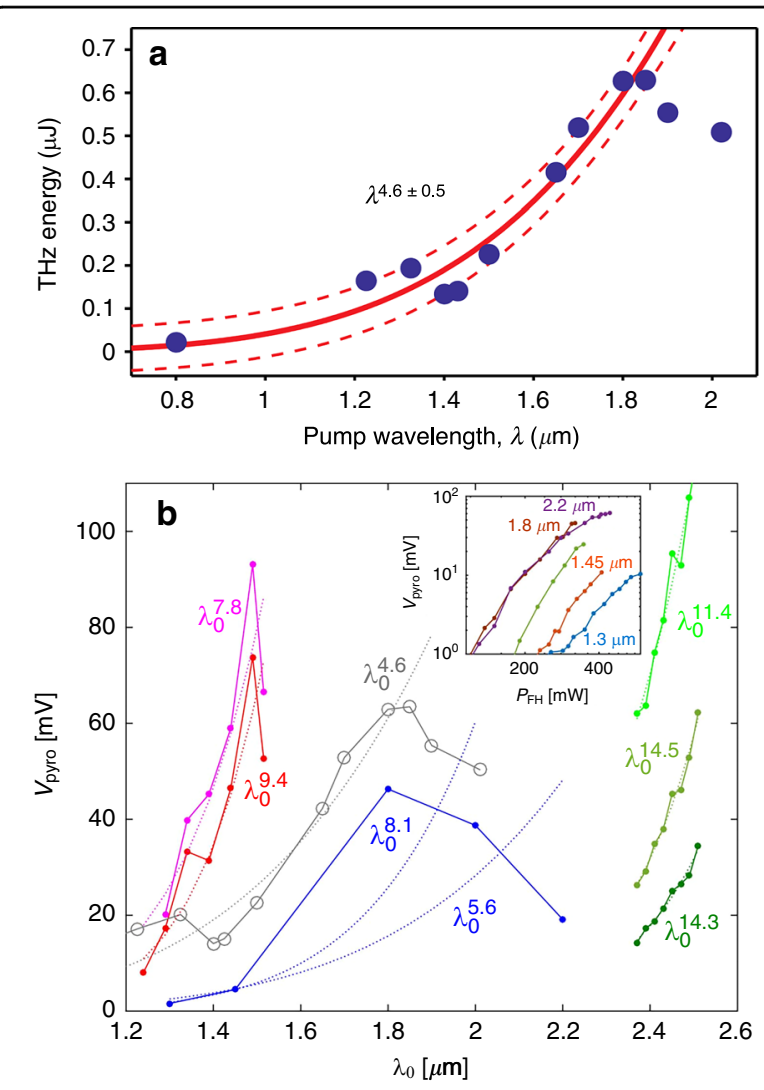

Fig. 7 Scaling of $\mathrm{THz}$ energy with the laser wavelength. a $\mathrm{THz}$ energy measured by Clerici et al. ${ }^{77}$ for 12 different pump wavelengths (solid circles). The red solid curve shows the power law fit $\lambda^{4.6 \pm 0.5}$ together with the $65 \%$ confidence bounds (red dashed curves). b Wavelength dependence of the pyroelectric detector signal indicating the $\mathrm{THz}$ energy yield in the experiments by Nguyen et al. ${ }^{78}$. Different point sets correspond to different OPAs and input pulse energies. The dotted curves provide the $\lambda^{a}$ fitting. The gray circles correspond to the data obtained by Clerici et al. ${ }^{77}$. Figure $\mathbf{a}$ is reprinted from ref. ${ }^{77}$ with permission from the American Physical Society, and b, from ref. ${ }^{78}$ with permission from the Optical Society of America

wavelengths from 0.8 to $2 \mu \mathrm{m}$, it was demonstrated that depending on the relative phase between the $\omega$ and $2 \omega$ pulse components, as well as their envelopes, the photocurrent model can justify powers $a$ between 2 and 5 . In turn, UPPE simulations of two-color filamentation performed in propagation geometry demonstrated $\lambda^{a}$ scaling of the THz energy yield with a power $a$ ranging from 2 to 3.5 .

The anomalous growth in $\mathrm{THz}$ energy with laser wavelength was specifically addressed in recent experiments by Nguyen et al $^{78}$, where two distinct optical parametric amplifiers (OPAs) were used to generate twocolor laser pulses with fundamental wavelengths from 1.2 to $2.6 \mu \mathrm{m}$. As a result, $\mathrm{THz}$ energies were measured to scale like $\lambda^{a}$ with large exponents $a$ ranging from 5.6 to
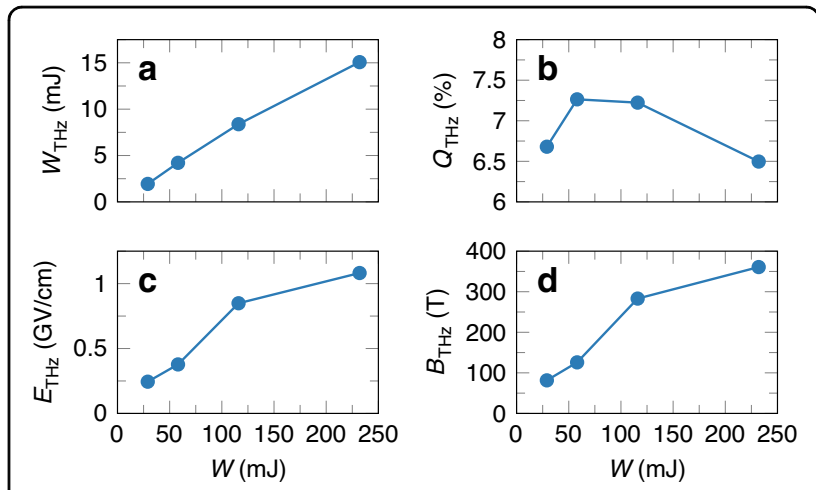

Fig. 8 Various parameters of $\mathrm{THz}$ radiation generated by midinfrared filaments. a THz pulse energy $W_{\mathrm{THz}}$ b THz conversion efficiency $Q_{T H z}$ c estimated peak electric $E_{T H z}$ and $\mathbf{d}$ magnetic field $B_{\mathrm{THz}}$ of a focused $\mathrm{THz}$ pulse versus the input energy $\mathrm{W}$ of $3.9 \mu \mathrm{m}$ twocolor laser pulses. Reprinted from ref. ${ }^{81}$ with permission from the American Physical Society

14.3 (see Fig. 7b). By means of comprehensive 3D simulations, Nguyen et al. demonstrated that these high scaling powers can be caused by variations in the laser beam size and pulse duration during the process of tuning the OPA carrier wavelength, as well as by subsequent deviations of the phase-matching conditions during the generation of the second harmonic component. In particular, it was shown that the lack of control of the phase difference between the $\omega$ and $2 \omega$ laser pulse components can explain the saturation of the $\mathrm{THz}$ energy yield at longer wavelengths.

We also would like to mention the experiments by Zhao et al. ${ }^{80}$, who studied two-color filamentation in different gases (helium, neon, argon, nitrogen, krypton, and xenon) at different wavelengths from 1.2 to $1.6 \mu \mathrm{m}$. The $\mathrm{THz}$ energy in these experiments was measured as a function of the input pulse energy, gas species, gas pressure, and pump wavelength. As a result, it was shown that stronger terahertz signals are more likely to be produced by longer wavelengths in heavier gases.

The above studies of two-color filamentation were carried out using typical OPAs as a source of the longwavelength laser pulses. Due to the restrictions of the OPA technique, the peak power of the obtained midinfrared laser pulses was less than the critical power $P_{\text {cr }}$ for Kerr self-focusing, thereby far from optimal for filamentation. Taking into account the appearance of new OPCPA laser systems capable of generating $3.9 \mu \mathrm{m}$ midinfrared laser pulses with a peak power exceeding $P_{c r}$, the $\mathrm{THz}$ generation by two-color filamentation in air at $3.9 \mu \mathrm{m}$ was compared to the case of $0.8 \mu \mathrm{m}$ in recent numerical studies by the present authors ${ }^{81,82}$. Figure 8 shows a brief summary of the obtained results. According to these results, with $3.9 \mu \mathrm{m}$ two-color laser pulses, one 
can achieve a $\mathrm{THz}$ conversion efficiency close to $7 \%$ (see Fig. 8b), which is more than two orders of magnitude higher than the $0.8 \mu \mathrm{m}$ case and appears to be the highest $\mathrm{THz}$ conversion efficiency reported to date compared to any of the known approaches for $\mathrm{THz}$ generation. It was shown that by further scaling of the input laser energy, it would be possible to generate multi-millijoule $\mathrm{THz}$ pulses (see Fig. 8a) with electric and magnetic fields at the GV/ $\mathrm{cm}$ and $\mathrm{kT}$ levels, respectively (see Fig. 8c, d). The high conversion efficiency of $\mathrm{THz}$ radiation driven by $3.9 \mu \mathrm{m}$ two-color laser pulses, in comparison with the nearinfrared ones, was explained by the synergy of the following factors: stronger photocurrents caused by higher ponderomotive forces, negligible walk-offs between harmonics, and longer and wider plasma channels. In addition, the authors predicted the existence of a novel mechanism in which higher harmonics generated in the process of two-color filamentation contribute to field symmetry breaking and thus enhance $\mathrm{THz}$ generation. The impressive increase in the $\mathrm{THz}$ conversion efficiency using $3.9 \mu \mathrm{m}$ two-color laser pulses in air was later confirmed by additional numerical simulations performed by Nguyen et al. ${ }^{83}$, who also considered the case of two-color filamentation at $10.6 \mu \mathrm{m}$.

To find an optimal laser wavelength for two-color filamentation in air, the whole range of possible two-color laser sources with fundamental wavelengths from 0.6 to $10.6 \mu \mathrm{m}$ was considered in a series of full 3D numerical simulations by the present authors ${ }^{84}$. For a fair comparison, the ratio of the laser pulse peak power to the critical power of self-focusing in these simulations was fixed at
1.2, independent of the wavelength. As a brief outcome of the obtained results, Fig. 9 shows how different parameters of the emitted $\mathrm{THz}$ radiation change with the wavelength. First, one can see that the $\mathrm{THz}$ energy grows non-monotonically with the wavelength, albeit without saturation (see Fig. 9a). Next, Fig. 9b shows that the THz conversion efficiency $Q_{\mathrm{THz}}$ depends on the wavelength $\lambda$ in a quite peculiar way, increasing and decreasing at wavelengths below $1.8 \mu \mathrm{m}$, then reaching the global maximum of approximately $7 \%$ at approximately $3.2 \mu \mathrm{m}$, and finally decreasing towards far-infrared wavelengths. The presence of the global maximum near $\lambda=3.2 \mu \mathrm{m}$ allows one to conclude that this laser wavelength is the optimal one for all $\mathrm{THz}$ sources based on two-color filamentation in air. In turn, Fig. 9c demonstrates that around $\lambda=4 \mu \mathrm{m}$, the initial growth of the $\mathrm{THz}$ field strength saturates at approximately $50 \mathrm{MV} / \mathrm{cm}$ within the filament. For applications, one can collect this $\mathrm{THz}$ radiation after the filament and refocus it, achieving electric field strengths at the $\mathrm{GV} / \mathrm{cm}$ level ${ }^{81,82}$. Finally, Fig. $9 \mathrm{~d}$ shows that the $\mathrm{THz}$ conical emission angle monotonically decreases with the wavelength, making the $\mathrm{THz}$ radiation from mid-infrared and far-infrared laser pulses better directed and easier to collect. As explained by the interference model $^{85-87}$, this effect is linked to the longer and thicker filament plasma channels generated by laser pulses with longer wavelengths.

The first experimental evidence of $\mathrm{THz}$ generation by $3.9 \mu \mathrm{m}$ two-color laser pulses was presented at the CLEO conference in $2018^{88}$. In these experiments, the authors used the high-power $3.9 \mu \mathrm{m}$ OPCPA laser system
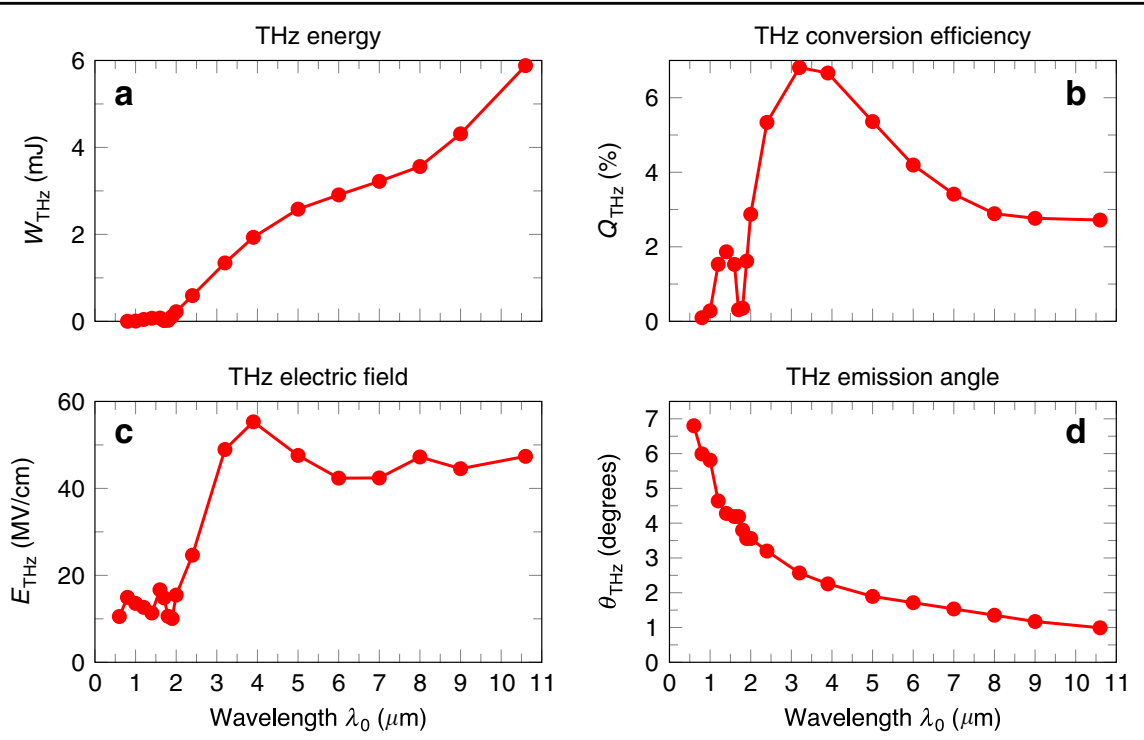

Fig. 9 Dependence of various THz parameters on the laser wavelength. a Energy of the generated THz pulse $W_{\text {THZ }} \mathbf{b}$ THz conversion efficiency $Q_{T H Z}$ c peak THz electric field $E_{T H z}$ and $\mathbf{d} T H z$ emission angle $\theta_{T H z}$ versus the wavelength $\lambda_{0}$ of the fundamental laser pulse. Reprinted from ${ }^{84}$ with permission from the Optical Society of America 

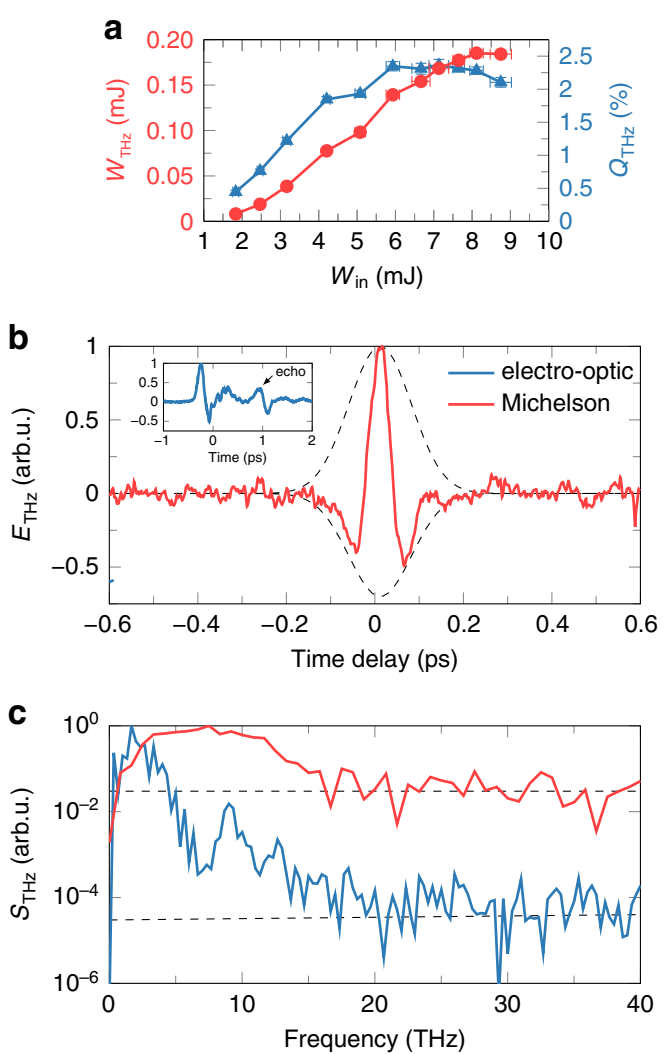

Fig. 10 Energy, temporal, and spectral characteristics of $\mathrm{THz}$ pulses generated by $3.9 \mu \mathrm{m}$ filaments. a Dependence of $\mathrm{THz}$ energy $W_{\mathrm{THz}}$ and the corresponding $\mathrm{THz}$ conversion efficiency $Q_{\mathrm{THz}}$ on the input laser energy $W_{\mathrm{in}}$. $\mathbf{b} \mathrm{THz}$ signals $E_{\mathrm{THz}}$ measured by electrooptic sampling (inset, blue) and by a Michelson interferometer (red). c THz power spectra $S_{\mathrm{THz}}$ (solid lines) obtained by the above techniques together with the noise levels of each technique (dashed lines). Reprinted from ref. ${ }^{90}$ under the terms of the Creative Commons CC BY license

developed at the TU Wien Photonics Institute ${ }^{32}$. However, due to the unoptimized experimental conditions, the maximum achieved $\mathrm{THz}$ energy at that time was only $\sim 49 \mu$, with the corresponding $\mathrm{THz}$ conversion efficiency being $\sim 0.77 \%$, which nonetheless was almost two orders of magnitude higher compared to the typical values reported for $0.8 \mu \mathrm{m}$ two-color laser pulses.

At the 2019 CLEO conference, the same group of authors presented the results of $\mathrm{THz}$ generation by $3.9 \mu \mathrm{m}$ two-color laser pulses obtained in a better optimized experiment on two-color filamentation ${ }^{89}$. Over the year, the energy of the emitted $\mathrm{THz}$ pulses increased up to $0.185 \mathrm{~mJ}$, and the $\mathrm{THz}$ conversion efficiency increased up to $2.36 \%$. The details of these experiments can be found in $^{90}$.

Figure 10 presents various parameters of the obtained $\mathrm{THz}$ pulses. In particular, Fig. 10a shows that with increasing input laser energy $W_{\text {in }}$, the energy of the $\mathrm{THz}$ pulses grows and reaches a maximum value of $0.185 \mathrm{~mJ}$ for $W_{\text {in }}$ equal to $8.12 \mathrm{~mJ}$. In turn, the corresponding maximum of the $\mathrm{THz}$ conversion efficiency reaches $2.36 \%$. At the same time, the measurements based on both electro-optic sampling and Michelson interferometry demonstrate that these high-energy $\mathrm{THz}$ pulses are single-cycle pulses with femtosecond duration and a spectral width of at least $20 \mathrm{THz}$ (see Fig. 10b, c). At this moment, the above values of the $\mathrm{THz}$ energy and $\mathrm{THz}$ conversion efficiency remain the highest among all values previously reported for plasma-based $\mathrm{THz}$ sources. Moreover, according to numerical simulations benchmarked by the experimental data, by further optimization of the existing experimental setup, it would be possible to significantly upscale the observed $\mathrm{THz}$ yield and reach a $\mathrm{THz}$ conversion efficiency of at least $\sim 5 \%{ }^{90}$.

Since then, two more experiments on two-color filamentation have demonstrated the significant enhancement of the THz yield for $3.9 \mu$ m laser pulses ${ }^{91,92}$. In both of them, $\mathrm{THz}$ radiation was studied as a part of the broadband supercontinuum generated by $3.9 \mu \mathrm{m}$ twocolor laser pulses jointly with the supercontinuum of higher harmonics. In the first experiment, conducted by Jang et al. ${ }^{91}$, the observed THz conversion efficiency was $\sim 1 \%$, with the corresponding $\mathrm{THz}$ energy being close to $30 \mu \mathrm{J}$. In addition, Jang et al. ${ }^{91}$ studied the dependence of the energy of $\mathrm{THz}$ pulses generated by $3.9 \mu \mathrm{m}$ laser pulses on the initial $\omega-2 \omega$ phase difference and found that it has the same $\pi / 2$ period as in the case of near-infrared laser pulses.

In the second experiment, carried out by Mitrofanov et al. ${ }^{92}$, THz generation by $3.9 \mu \mathrm{m}$ two-color laser pulses was studied in various gases (helium, argon, nitrogen, air, and krypton) under different pressures. Gases with a lower ionization potential were shown to provide a higher $\mathrm{THz}$ yield. In particular, under the fixed energy of the $3.9 \mu \mathrm{m}$ laser pulse, the highest $\mathrm{THz}$ energy $(24 \mu \mathrm{J})$ was achieved in the experiments with krypton. For comparison, the highest $\mathrm{THz}$ energy measured in air was $18 \mu \mathrm{J}$ (corresponding to a $5 \mathrm{MV} / \mathrm{cm} \mathrm{THz}$ field after focusing).

In Table 1 , we summarize the parameters of the $\mathrm{THz}$ pulses generated in experiments using two-color filamentation with different laser wavelengths. The existing mid-infrared laser sources are clearly able to generate $\mathrm{THz}$ fields with energies and amplitudes exceeding, respectively, $0.1 \mathrm{~mJ}$ and $0.1 \mathrm{GV} / \mathrm{cm}$. According to the theoretical predictions, in the near future, we can expect the appearance of $\mathrm{THz}$ sources capable of generating multi-millijoule $\mathrm{THz}$ pulses with peak $\mathrm{THz}$ electric fields at the $\mathrm{GV} / \mathrm{cm}$ level. To gain some intuition about the strength of these $\mathrm{THz}$ fields, let us consider them in the context of current THz-based electron accelerators. Since the electron energy gain and acceleration gradient scale 
Table 1 Energy $W_{\mathrm{THz}}$, conversion efficiency $Q_{\mathrm{THz}}$ and peak electric field $E_{\mathrm{THz}}$ of $\mathrm{THz}$ pulses generated in experiments using two-color filamentation with different laser wavelengths $\lambda$

\begin{tabular}{llll}
\hline $\boldsymbol{\lambda}(\boldsymbol{\mu m})$ & $\boldsymbol{W}_{\mathrm{THz}}(\boldsymbol{\mu})$ & $\boldsymbol{Q}_{\mathrm{THz}}(\%)$ & $E_{\mathrm{THz}}$ Ref. $(\mathbf{M V} / \mathrm{cm})$ \\
\hline 0.8 & 1.44 & 0.01 & $8^{17}$ \\
0.8 & 31 & 0.07 & $21^{18}$ \\
1.5 & 0.099 & 0.56 & $-{ }^{80}$ \\
1.85 & 0.63 & 0.16 & $4.4^{77}$ \\
3.9 & 18 & 0.3 & $5^{92}$ \\
3.9 & 27 & 1 & $-{ }^{91}$ \\
3.9 & 185 & 2.36 & $150^{90}$ \\
\hline
\end{tabular}

linearly with the $\mathrm{THz}$ field amplitude, one would expect $\mathrm{GV} / \mathrm{cm} \mathrm{THz}$ fields to allow achieving multi-GeV/m gradients and electron energy gains exceeding $10 \mathrm{MeV}-$ numbers that are characteristic of electron accelerators based on petawatt laser technologies ${ }^{7,8}$.

\section{Conclusions}

In summary, we have reviewed the most promising way to date of developing high-peak-power $\mathrm{THz}$ sources. $\mathrm{THz}$ sources driven by ultrashort mid-infrared and far-infrared two-color laser filamentation demonstrate unprecedented conversion efficiencies on the level of 5-7\%. Starting from the fundamental microscopic physical mechanisms, we drove all the way to nonlinear propagation in the form of filaments and explained the physics behind these sources. We showed that although some aspects of the fundamental processes have still not been fully elucidated, such as the role of many-body effects, quantitative agreement between the theory and the experiments can be achieved if one accounts carefully for the complex spatiotemporal phenomena of two-color filamentation.

The development of powerful $\mathrm{THz}$ sources similar to the ones we discussed here opens up new horizons in $\mathrm{THz}$ nonlinear science. The experimental findings and the most recent numerical simulations we discussed here point to a new generation of extreme-power $\mathrm{THz}$ transients. Near-future THz sources, driven by two-color midinfrared and far-infrared filaments, are projected to offer multi-millijoule energies per pulse and peak electric and magnetic fields at the gigavolt per centimeter and kilotesla levels, respectively. Quasi-static ultrashort electric and magnetic bursts at these intensities will enable free space extreme nonlinear and relativistic science for not only accessing unexplored basic science problems but also offering unique solutions to demanding applications, such as compact charged particle accelerators.

\section{Acknowledgements}

This work was supported by the National Priorities Research Program grant No. NPRP1 1S-1128-170042 from the Qatar National Research Fund (member of The Qatar Foundation), the H2020 Laserlab-Europe (EC-GA 871124), the H2020 MIRBOSE (EC-GA 737017), and the "HELLAS-CH" (MIS 5002735), co-financed by Greece and the European Union. We also thank Anastasios D. Koulouklidis for the preparation of Fig. 1 with the typical setup for THz generation.

\section{Author details}

${ }^{1}$ Science Program, Texas A\&M University at Qatar, P.O. Box 23874, Doha, Qatar. ${ }^{2}$ P.N. Lebedev Physical Institute of the Russian Academy of Sciences, 53 Leninskiy Prospekt, Moscow 119991, Russia. ${ }^{3}$ Institute of Electronic Structure and Laser (IESL), Foundation for Research and Technology-Hellas (FORTH), P.O. Box 1527, Heraklion GR-71110, Greece. ${ }^{4}$ Department of Materials Science and Technology, University of Crete, Heraklion GR-71003, Greece

Conflict of interest

The authors declare that they have no conflict of interest.

Received: 13 May 2020 Revised: 11 October 2020 Accepted: 19 October 2020

Published online: 12 November 2020

References

1. Tonouchi, M. Cutting-edge terahertz technology. Nat. Photon. 1, 97-105 (2007).

2. Danciu, M. et al. Terahertz spectroscopy and imaging: a cutting-edge method for diagnosing digestive cancers. Materials 12, 1519 (2019).

3. Massaouti, M. et al. Detection of harmful residues in honey using terahertz time-domain spectroscopy. Appl. Spectrosc. 67, 1264-1269 (2013).

4. Manceau, J.-M. et al. Terahertz time domain spectroscopy for the analysis of cultural heritage related materials. Appl. Phys. B 90, 365-368 (2008).

5. Kampfrath, T., Tanaka, K. \& Nelson, K. A. Resonant and nonresonant control over matter and light by intense terahertz transients. Nat. Photon. 7, 680-690 (2013).

6. Mittleman, D. M. Perspective: terahertz science and technology. J. Appl. Phys. 122, 230901 (2017).

7. Nanni, E. A. et al. Terahertz-driven linear electron acceleration. Nat. Commun. $\mathbf{6}$, 8486 (2015).

8. Curry, E. et al. Meter-scale terahertz-driven acceleration of a relativistic beam. Phys. Rev. Lett. 120, 094801 (2018).

9. Zhang, D. F. et al. Segmented terahertz electron accelerator and manipulator (STEAM). Nat. Photon. 12, 336-342 (2018).

10. Balogh, E. et al. Single attosecond pulse from terahertz- assisted high-order harmonic generation. Phys. Rev. A 84, 023806 (2011).

11. Tóth, G. et al. Single-cycle attosecond pulses by Thomson backscattering of terahertz pulses. J. Optical Soc. Am. B 35, A103-A109 (2018).

12. Fülöp, J. A., Tzortzakis, S. \& Kampfrath, T. Laser-driven strong-field terahertz sources. Adv. Optical Mater. 8, 1900681 (2020).

13. Fülöp, J. A. et al. Efficient generation of $\mathrm{THz}$ pulses with $0.4 \mathrm{~mJ}$ energy. Opt. Express 22, 20155-20163 (2014).

14. Vicario, C. et al. Generation of 0.9-mJ THz pulses in DSTMS pumped by a $\mathrm{Cr}$ : $\mathrm{Mg}_{2} \mathrm{SiO}_{4}$ laser. Opt. Lett. 39, 6632-6635 (2014).

15. Shalaby, M. \& Hauri, C. P. Demonstration of a low- frequency threedimensional terahertz bullet with extreme brightness. Nat. Commun. 6, 5976 (2015).

16. Kim, K. Y. et al. Coherent control of terahertz supercontinuum generation in ultrafast laser-gas interactions. Nat. Photon. 2, 605-609 (2008).

17. Oh, T. I. et al. Generation of strong terahertz fields exceeding $8 \mathrm{MV} / \mathrm{cm}$ at 1 kHz and real-time beam profiling. Appl. Phys. Lett. 105, 041103 (2014).

18. Kuk, D. et al. Generation of scalable terahertz radiation from cylindrically focused two-color laser pulses in air. Appl. Phys. Lett. 108, 121106 (2016).

19. Jin, Q. et al. Observation of broadband terahertz wave generation from liquid water. Appl. Phys. Lett. 111, 071103 (2017).

20. Dey, I. et al. Highly efficient broadband terahertz generation from ultrashort laser filamentation in liquids. Nat. Commun. 8, 1184 (2017). 
21. Kim, K.-Y. et al. Terahertz emission from ultrafast ionizing air in symmetrybroken laser fields. Opt. Express 15, 4577-4584 (2007).

22. Huang, W. R. et al. Highly efficient terahertz pulse generation by optical rectification in stoichiometric and cryo-cooled congruent lithium niobate. J. Mod. Opt. 62, 1486-1493 (2015).

23. Matsubara, E., Nagai, M. \& Ashida, M. Ultrabroadband coherent electric field from far infrared to $200 \mathrm{THz}$ using air plasma induced by $10 \mathrm{fs}$ pulses. Appl. Phys. Lett. 101, 011105 (2012).

24. Oh, T. I. et al. Intense terahertz generation in two-color laser filamentation: energy scaling with terawatt laser systems. N. J. Phys. 15, 075002 (2013).

25. Wang, T.-J. et al. Toward remote high energy terahertz generation. Appl. Phys. Lett. 97, 111108 (2010).

26. Wang, T.-J. et al. Remote generation of high-energy terahertz pulses from two-color femtosecond laser filamentation in air. Phys. Rev. A 83, 053801 (2011).

27. Daigle, J.-F. et al. Remote THz generation from two-color filamentation: long distance dependence. Opt. Express 20, 6825-6834 (2012).

28. Liu, K. et al. Enhanced terahertz wave emission from air-plasma tailored by abruptly autofocusing laser beams. Optica 3, 605-608 (2016).

29. Zhong, H., Karpowicz, N. \& Zhang, X. C. Terahertz emission profile from laserinduced air plasma. Appl. Phys. Lett. 88, 261103 (2006).

30. Klarskov, P. et al. Experimental three-dimensional beam profiling and modeling of a terahertz beam generated from a two-color air plasma. N. J. Phys. 15, 075012 (2013).

31. Blank, V., Thomson, M. D. \& Roskos, H. G. Spatio-spectral characteristics of ultrabroadband thz emission from two-colour photoexcited gas plasmas and their impact for nonlinear spectroscopy. N. J. Phys. 15, 075023 (2013).

32. Andriukaitis, G. et al. $90 \mathrm{GW}$ peak power few-cycle mid-infrared pulses from an optical parametric amplifier. Opt. Lett. 36, 2755-2757 (2011).

33. Mitrofanov, A. V. et al. Mid-infrared laser filaments in the atmosphere. Sci. Rep. 5, 8368 (2015).

34. Shumakova, V. et al. Multi-millijoule few-cycle mid-infrared pulses through nonlinear self-compression in bulk. Nat. Commun. 7, 12877 (2016).

35. Haberberger, D., Tochitsky, S. \& Joshi, C. Fifteen terawatt picosecond $\mathrm{CO}_{2}$ laser system. Opt. Express 18, 17865-17875 (2010).

36. Polyanskiy, M. N., Babzien, M. \& Pogorelsky, I. V. Chirped-pulse amplification in a $\mathrm{CO}_{2}$ laser. Optica 2, 675-681 (2015).

37. Zheltikov, A. M. Laser-induced filaments in the mid-infrared. J. Phys. B 50, 092001 (2017).

38. Tochitsky, S. et al. Filamentation of long-wave infrared pulses in the atmosphere [Invited]. J. Optical Soc. Am. B 36, G40-G41 (2019).

39. Couairon, A. \& Mysyrowicz, A. Femtosecond filamentation in transparent media. Phys. Rep. 441, 47-189 (2007).

40. Keldysh, L. V. Ionization in the field of a strong electromagnetic wave. Sov. J. Exp. Theor. Phys. 20, 1307-1314 (1965).

41. Babushkin, I. et al. Terahertz and higher-order Brunel harmonics: from tunnel to multiphoton ionization regime in tailored fields. J. Mod. Opt. 64, 1078-1087 (2017).

42. Agostini, P. \& DiMauro, L. F. Atoms in high intensity mid-infrared pulses. Contemp. Phys. 49, 179-197 (2008).

43. Becker, W. et al. The plateau in above-threshold ionization: the keystone of rescattering physics. J. Phys. B 51, 162002 (2018).

44. Blaga, C. I. et al. Strong-field photoionization revisited. Nat. Phys. 5, 335-338 (2009).

45. Quan, W. et al. Classical aspects in above-threshold ionization with a midinfrared strong laser field. Phys. Rev. Lett. 103, 093001 (2009).

46. Huang, Y. D. et al. High-harmonic and terahertz spectroscopy (HATS): methods and applications. Appl. Sci. 9, 853 (2019).

47. Serebryannikov, E. E. \& Zheltikov, A. M. Quantum and semiclassical physics behind ultrafast optical nonlinearity in the midinfrared: the role of ionization dynamics within the field half cycle. Phys. Rev. Lett. 113, 043901 (2014).

48. Lai, Y. H. et al. Experimental investigation of strong-field-ionization theories for laser fields from visible to midinfrared frequencies. Phys. Rev. A 96, 063417 (2017).

49. Perelomov, A., Popov, V. \& Terent'ev, M. Ionization of atoms in an alternating electric field. Sov. J. Exp. Theor. Phys. 23, 924-934 (1966).

50. Popruzhenko, S. V. et al. Strong field ionization rate for arbitrary laser frequencies. Phys. Rev. Lett. 101, 193003 (2008).

51. Schuh, $\mathrm{K}$. et al. Influence of many-body interactions during the ionization of gases by short intense optical pulses. Phys. Rev. E 89, 033103 (2014).
52. Schuh, K. et al. Influence of optical and interaction-induced dephasing effects on the short-pulse ionization of atomic gases. J. Optical Soc. Am. B 32 , 1442-1449 (2015)

53. Schuh, K. Moloney, J. V. \& Koch, S. W. Interaction-induced nonlinear refractiveindex reduction of gases in the midinfrared regime. Phys. Rev. E 93, 013208 (2016).

54. Schuh, K. et al. Self-channeling of high-power long-wave infrared pulses in atomic gases. Phys. Rev. Lett. 118, 063901 (2017).

55. Tochitsky, S. et al. Megafilament in air formed by self-guided terawatt longwavelength infrared laser. Nat. Photon. 13, 41-46 (2019).

56. Woodbury, D. et al. Absolute measurement of laser ionization yield in atmospheric pressure range gases over 14 decades. Phys. Rev. Lett. 124 013201 (2020).

57. Woodbury, D. et al. Self-guiding of long-wave infrared laser pulses mediated by avalanche ionization. Phys. Rev. Lett. 125, 133201 (2020).

58. Wright, E. M. et al. Memory effects in the long-wave infrared avalanche ionization of gases: a review of recent progress. Rep. Prog. Phys. 82, 064401 (2019).

59. Gao, X. H. \& Shim, B. Impact-ionization mediated self-focusing of longwavelength infrared pulses in gases. Opt. Lett. 44, 827-830 (2019).

60. Mlejnek, M., Wright, E. M. \& Moloney, J. V. Dynamic spatial replenishment of femtosecond pulses propagating in air. Opt. Lett. 23, 382-384 (1998).

61. Langevin, D. et al. Determination of molecular contributions to the nonlinear refractive index of air for mid-infrared femtosecond laser-pulse excitation. Phys. Rev. A 99, 063418 (2019).

62. Zahedpour, S., Wahlstrand, J. K. \& Milchberg, H. M. Measurement of the nonlinear refractive index of air constituents at mid-infrared wavelengths. Opt. Lett. 40, 5794-5797 (2015).

63. Zahedpour, S., Hancock, S. W. \& Milchberg, H. M. Ultrashort infrared 2.5-11 $\mu \mathrm{m}$ pulses: spatiotemporal profiles and absolute nonlinear response of air constituents. Opt. Lett. 44, 843-846 (2019).

64. Brown, J. M., Couairon, A. \& Gaarde, M. B. Ab initio calculations of the linear and nonlinear susceptibilities of $\mathrm{N}_{2}, \mathrm{O}_{2}$, and air in midinfrared laser pulses. Phys. Rev. A 97, 063421 (2018).

65. Wahlstrand, J. K. et al. Bound-electron nonlinearity beyond the ionization threshold. Phys. Rev. Lett. 120, 183901 (2018).

66. Pigeon, J. J. et al. Measurements of the nonlinear refractive index of air, $\mathrm{N}_{2}$, and $\mathrm{O}_{2}$ at $10 \mu \mathrm{m}$ using four-wave mixing. Opt. Lett. 41, 3924-3927 (2016).

67. Pigeon, J. J. et al. Experimental study of the third-order nonlinearity of atomic and molecular gases using 10- $\mu$ m laser pulses. Phys. Rev. A 97, 043829 (2018).

68. HITRAN on the Web. (2020-02-04). http://hitran.iao.ru/. (2020).

69. Panov, N. A. et al. Supercontinuum of a 3.9- $\mu \mathrm{m}$ filament in air: formation of a two-octave plateau and nonlinearly enhanced linear absorption. Phys. Rev. A 94, 041801(R) (2016).

70. Kompanets, V. O. et al. Nonlinear enhancement of resonance absorption at the filamentation of a mid-infrared pulse in high-pressure gases. JETP Lett. 111, 31-35 (2020).

71. Whalen, P. et al. Extreme carrier shocking of intense long-wavelength pulses. Phys. Rev. A 89, 023850 (2014).

72. Panagiotopoulos, P. et al. Super high power mid-infrared femtosecond light bullet. Nat. Photon. 9, 543-548 (2015)

73. Hofstrand, A. \& Moloney, J. V. Optical carrier-wave sub-cycle structures associated with supercritical collapse of long-wavelength intense pulses propagating in weakly anomalously dispersive media. Phys. Rev. Lett. 124, 043901 (2020).

74. Zhokhov, P. A. \& Zheltikov, A. M. Attosecond shock waves. Phys. Rev. Lett. 110 183903 (2013).

75. Wang, W.-M. et al. Efficient terahertz emission by mid-infrared laser pulses from gas targets. Opt. Lett. 36, 2608-2610 (2011).

76. Bergé, L. et al. Numerical simulations of $\mathrm{THz}$ generation by two-color laser filaments. Phys. Rev. Lett. 110, 073901 (2013).

77. Clerici, M. et al. Wavelength scaling of terahertz generation by gas ionization. Phys. Rev. Lett. 110, 253901 (2013).

78. Nguyen, A. et al. Wavelength scaling of terahertz pulse energies delivered by two-color air plasmas. Opt. Lett. 44, 1488-1491 (2019).

79. Nguyen, A. et al. Spectral dynamics of $\mathrm{THz}$ pulses generated by two-color laser filaments in air: the role of Kerr nonlinearities and pump wavelength. Opt. Express 25, 4720 (2017).

80. Zhao, H. et al. Terahertz wave generation from noble gas plasmas induced by a wavelength-tunable femtosecond laser. IEEE Trans. Terahertz Sci. Technol. 8, 299-304 (2018)

81. Fedorov, V. Y. \& Tzortzakis, S. Extreme THz fields from two-color filamentation of midinfrared laser pulses. Phys. Rev. A 97, 063842 (2018). 
82. Fedorov, V. Y. \& Tzortzakis, S. Extreme THz fields from two-color filamentation of mid-infrared laser pulses. Preprint at arXiv https:/arxiv.org/abs/1708.07310 (2017).

83. Nguyen, A. et al. Broadband terahertz radiation from two-color mid- and farinfrared laser filaments in air. Phys. Rev. A 97, 063839 (2018).

84. Fedorov, V. Y. \& Tzortzakis, S. Optimal wavelength for two-color filamentationinduced terahertz sources. Opt. Express 26, 31150-31159 (2018).

85. You, Y. S., Oh, T. I. \& Kim, K. Y. Off-axis phase-matched terahertz emission from two-color laser-induced plasma filaments. Phys. Rev. Lett. 109, 183902 (2012).

86. Gorodetsky, A. et al. Physics of the conical broadband terahertz emission from two-color laser-induced plasma filaments. Phys. Rev. A 89, 033838 (2014).

87. Zhang, Z. L. et al. Controllable terahertz radiation from a linear-dipole array formed by a two-color laser filament in air. Phys. Rev. Lett. 117, 243901 (2016).
88. Koulouklidis, A. D. et al. Observation of strong $\mathrm{THz}$ fields from mid-infrared two-color laser filaments. Conference on Lasers and Electro-Optics. (OSA, San Jose, 2018).

89. Koulouklidis, A. D. et al. Two-color mid-infrared laser filaments produce terahertz pulses with extreme efficiency. In 2019 Conference on Lasers and ElectroOptics Europe \& European Quantum Electronics Conference (CLEO/Europe-EQEC) (IEEE, Munich, 2019).

90. Koulouklidis, A. D. et al. Observation of extremely efficient terahertz generation from mid-infrared two- color laser filament. Nat. Commun. 11, 292 (2020).

91. Jang, D. et al. Efficient terahertz and Brunel harmonic generation from air plasma via mid-infrared coherent control. Optica 6, 1338-1341 (2019).

92. Mitrofanov, A. V. et al. Ultraviolet-to-millimeter-band supercontinua driven by ultrashort mid-infrared laser pulses. Optica 7, 15-19 (2020). 